\title{
FORMALIZED LINEAR ALGEBRA OVER ELEMENTARY DIVISOR RINGS IN COQ
}

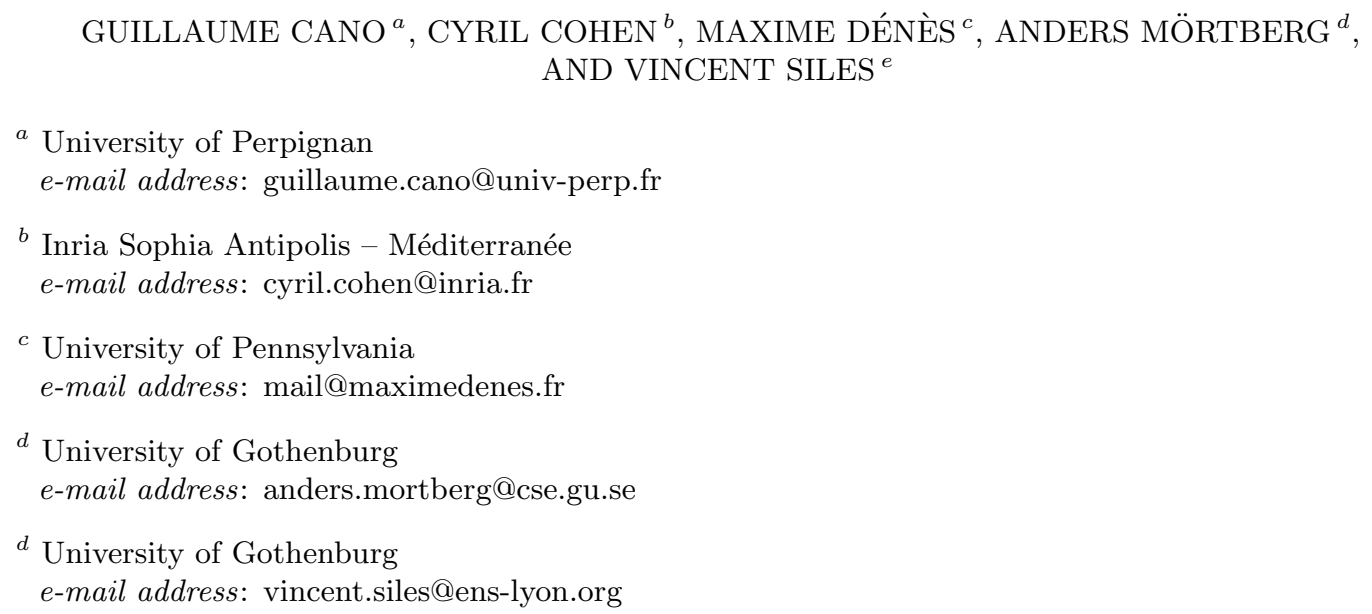

\begin{abstract}
This paper presents a COQ formalization of linear algebra over elementary divisor rings, that is, rings where every matrix is equivalent to a matrix in Smith normal form. The main results are the formalization that these rings support essential operations of linear algebra, the classification theorem of finitely presented modules over such rings and the uniqueness of the Smith normal form up to multiplication by units. We present formally verified algorithms computing this normal form on a variety of coefficient structures including Euclidean domains and constructive principal ideal domains. We also study different ways to extend Bézout domains in order to be able to compute the Smith normal form of matrices. The extensions we consider are: adequacy (i.e. the existence of a gdco operation), Krull dimension $\leq 1$ and well-founded strict divisibility.
\end{abstract}

\section{INTRODUCTION}

The goal of this paper is to develop linear algebra for elementary divisor rings, that is, rings where there is an algorithm for computing the Smith normal form of matrices. More specifically, we focus on the axiomatics and basic algorithms of such rings. This work fits within a bigger project of formalizing theories and algorithms for constructive module theory. This lays ground to program more efficient algorithms (like in Axiom, MAPle, Magma, ...), and prove them correct with regard to the notions introduced here, using the CoQEAL methodology [12, 5], developed by some of the authors (cf. section 7).

2012 ACM CCS: [Theory of computation]: Logic-Logic and verification / Constructive mathematics / Type theory.

Key words and phrases: Formalization of mathematics, Constructive algebra, CoQ, SSREFLECT.

L而

드 LOGICAL METHODS IN COMPUTER SCIENCE
DOI:10.2168/LMCS-12(2:7)2016
(C) G. Cano, C. Cohen, M. Dénès, A. Mörtberg, and V. Siles (C) Creative Commons 
In this paper, we do not contribute with new theorems or new efficient algorithms in the field, except for some minor factoring in order to simplify the formal proof. However, we provide a formal framework to develop more theory and more complex algorithms in CoQ, by regrouping and representing folklore concepts in the proof assistant, that we found scattered in the literature. We make a synthesis of different axiomatics and show how they are linked constructively. In order to do that, we first introduce the classical notions at use and their constructive variants (section 2).

The main contributions of this paper are the formalization 1 , using the CoQ proof assistant with the SSREFLECT extension, of:

- rings with explicit divisibility, GCD domains, Bézout domains, constructive principal ideal domains and Euclidean domains (section 3), which corresponds to the file theory/dvdring.v;

- an algorithm computing the Smith normal form of matrices with coefficients in Euclidean domains (files theory/dvdring.v and refinements/smith.v) and the generalization to constructive principal ideal domains (section 4), which corresponds to the file refinements/smithpid.v;

- linear algebra over elementary divisor rings together with a proof that the Smith normal form is unique up to multiplication by units for rings with a gcd operation (section 5) (file theory/edr.v) and the classification theorem for finitely presented modules over elementary divisor rings (file theory/fpmod.v); and

- proofs that Bézout domains extended with one of the three extensions above are elementary divisor rings and how these notions are related (section 6) which corresponds to the file theory/kaplansky.v.

The paper ends with an overview of related work (section 7), followed by conclusions and future work (section 8).

\section{Classical axiomatics and COnstructive variants}

The algorithms we present for computing the Smith normal form can be seen as generalizations of Gaussian elimination that can, in particular, be defined for $\mathbb{Z}$. The main source of inspiration for this work is the formalization of finite dimensional vector spaces by Georges Gonthier [17] in which spaces are represented using matrices and all subspace constructions can be elegantly defined from Gaussian elimination. This enables a concrete and point-free presentation of linear algebra which is suitable for formalization as it takes advantage of the small scale reflection methodology of the SSREFLECT extension and the Mathematical Components library [18] for the CoQ proof assistant [8]. When generalizing this to elementary divisor rings there are two essential problems that need to be resolved before the theory may be formalized:

(1) What is a suitable generalization of finite dimensional vector spaces when considering more general classes of rings than fields as coefficients?

(2) What rings are elementary divisor rings?

A possible answer to the first problem is finitely generated $R$-modules, i.e. finite dimensional vector spaces with coefficients in a general ring instead of a field. However these are not as well behaved as finite dimensional vector spaces as there might be relations among the

\footnotetext{
${ }^{1}$ The formal development is a subset of the repository: https://github.com/CoqEAL/CoqEAL and the companion material for this paper has been regrouped here: http://www.cyrilcohen.fr/work/edr/
} 
generators. In other words, not all finitely generated modules are free. To overcome this, we restrict our attention further and consider finitely presented modules, which are modules specified by a finite number of generators and a finite number of relations between these. This class of modules may be represented concretely using matrices, which in turn means that we can apply the same approach as in [17] and implement all operations by manipulating the presentation matrices.

A standard answer to the second problem is principal ideal domains like $\mathbb{Z}$ and the ring of univariate polynomials over a field (denoted by $k[x]$ ). The classical definition of principal ideal domains is integral domains where all ideals are principal (i.e. generated by one element). In particular it means that principal ideal domains are Noetherian as all ideals are finitely generated. Classically this is equivalent to the ascending chain condition for ideals, however in order to prove this equivalence classical reasoning is used in essential ways. In fact, if these definitions are read constructively they are so strong that no ring except the trivial ring satisfies them [33]. Principal ideal domains are hence problematic from a constructive point of view as they are Noetherian.

A possible solution is to restrict the attention to Euclidean domains (which include both $\mathbb{Z}$ and $k[x])$ and show how to compute the Smith normal form of matrices over these rings. This approach is appealing as it allows for a simple definition of the Smith normal form algorithm that resembles the one of Gaussian elimination. While Euclidean domains are important, we would like to be more general. In order to achieve this we consider an alternative approach that is customary in constructive algebra: to generalize all statements and not assume Noetheriannity at all [26. If we do this for principal ideal domains we get Bézout domains, which are rings where every finitely generated ideal is principal. However, it is an open problem whether all Bézout domains are elementary divisor rings or not [27]. Hence we study different assumptions that we can add to Bézout domains in order to prove that they are elementary divisor rings. The properties we define and study independently are:

(1) Adequacy (i.e. the existence of a gdco operation);

(2) Krull dimension $\leq 1$;

(3) Strict divisibility is well-founded.

The last one can be seen as a constructive approximation to the ascending chain condition for principal ideals, so this kind of Bézout domains will be referred to as constructive principal ideal domains.

\section{Rings With EXPLICIT DIVISIBILITY}

In this section we recall definitions and basic properties of rings with explicit divisibility, GCD domains, Bézout domains, constructive principal ideal domains and Euclidean domains.

3.1. Rings with explicit divisibility. Throughout the paper all rings are discrete integral domains, i.e. commutative rings with a unit, decidable equality and no zero divisors. This section is loosely based on the presentation of divisibility in discrete domains of Mines, Richman and Ruitenberg in [30. The central notion we consider is:

Definition 3.1. A ring $R$ has explicit divisibility if it has a divisibility test that produces witnesses. 
That is, given $a$ and $b$ we can test if $a \mid b$ and if this is the case get $x$ such that $b=x a$. Two elements $a, b \in R$ are associates if $a \mid b$ and $b \mid a$, which is equivalent to $b=u a$ for some unit $u$ because we have cancellation. Note that this gives rise to an equivalence relation. This notion will play an important role later as we will show that the Smith normal form of a matrix is unique up to multiplication by units, that is, up to associated elements.

A GCD domain is an example of a ring with explicit divisibility:

Definition 3.2. A GCD domain $R$ is a ring with explicit divisibility in which every pair of elements has a greatest common divisor, that is, for $a, b \in R$ there is $\operatorname{gcd}(a, b)$ such that $\operatorname{gcd}(a, b)|a, \operatorname{gcd}(a, b)| b$ and $\forall g,(g \mid a) \wedge(g \mid b) \rightarrow g \mid \operatorname{gcd}(a, b)$.

Note first that we make no restriction on $a$ and $b$, so they can both be zero. In this case the greatest common divisor is zero. This makes sense as zero is the maximum element for the divisibility relation. Note also that as $R$ is assumed to be a ring with explicit divisibility we get that $\operatorname{gcd}(a, b) \mid a$ means that there is $a^{\prime}$ such that $a=a^{\prime} \operatorname{gcd}(a, b)$. By Euclid's algorithm we know that both $\mathbb{Z}$ and $k[x]$ are GCD domains.

With the above definition the greatest common divisor of two elements is not necessarily unique, e.g. the greatest common divisor of 2 and 3 in $\mathbb{Z}$ is either 1 or -1 . But if we consider equality up to multiplication by units (i.e. up to associatedness) the greatest common divisor is unique, so in the rest of the paper equality will denote equality up to associatedness when talking about the gcd.

Most of the rings we will study in this paper are Bézout domains:

Definition 3.3. A Bézout domain is a GCD domain $R$ such that for any two elements $a, b \in R$ there is $x, y \in R$ such that $a x+b y=\operatorname{gcd}(a, b)$.

Let $a$ and $b$ be two elements in a ring $R$. If $R$ is a GCD domain we can compute $g=\operatorname{gcd}(a, b)$ together with witnesses to the ideal inclusion $(a, b) \subseteq(g)$. Further, if $R$ is a Bézout domain we can compute witnesses for the inclusion $(g) \subseteq(a, b)$ as well. This can be generalized to multiple elements $a_{1}, \ldots, a_{n} \in R$ to obtain witnesses for the inclusions $\left(a_{1}, \ldots, a_{n}\right) \subseteq(g)$ and $(g) \subseteq\left(a_{1}, \ldots, a_{n}\right)$ where $g$ is the greatest common divisor of the $a_{i}$. Bézout domains can hence be characterized as rings in which every finitely generated ideal is principal, which means that they are non-Noetherian generalizations of principal ideal domains.

Note that, on the one hand there exists $a^{\prime}$ and $b^{\prime}$ such that $a=a^{\prime} g$ and $b=b^{\prime} g$, and on the other hand we have $x$ and $y$ such that $a x+b y=g$. Therefore, by dividing with $g$, we obtain a Bézout relation between $a^{\prime}$ and $b^{\prime}$, namely $a^{\prime} x+a^{\prime} y=1$.

This definition can be extended to give a constructive version of principal ideal domains. We say that $a$ divides $b$ strictly if $a \mid b$ but $b \nmid a$, then we can define:

Definition 3.4. A constructive principal ideal domain is a Bézout domain in which the strict divisibility relation is well-founded.

By well-founded we mean that any descending chain of strict divisions is finite. This can be seen as a constructive approximation to the ascending chain condition for principal ideals and hence to Noetheriannity. Both $\mathbb{Z}$ and $k[x]$ can be proved to be Bézout domains and satisfy the condition of constructive principal ideal domains. In fact, this can be done for any ring on which the extended Euclidean algorithm can be implemented. These rings are called Euclidean domains: 
Definition 3.5. A Euclidean domain is a ring $R$ with a Euclidean norm $\mathcal{N}: R \rightarrow \mathbb{N}$ such that for any $a \in R$ and nonzero $b \in R$ we have $\mathcal{N}(a) \leqslant \mathcal{N}(a b)$. Further, for any $a \in R$ and nonzero $b \in R$ we can find $q, r \in R$ such that $a=b q+r$ and either $r=0$ or $\mathcal{N}(r)<\mathcal{N}(b)$.

In the case of $\mathbb{Z}$ and $k[x]$ we can take respectively the absolute value function and the degree function as Euclidean norm. Then the standard division algorithms for these rings can be used to compute $q$ and $r$.

3.2. Formalization of algebraic structures. The algebraic structures have been formalized in the same manner as in the SSREFLECT library [16] using packed classes (implemented by mixins and canonical structures). We will now discuss the formalization of these new structures starting with the definition of rings with explicit divisibility:

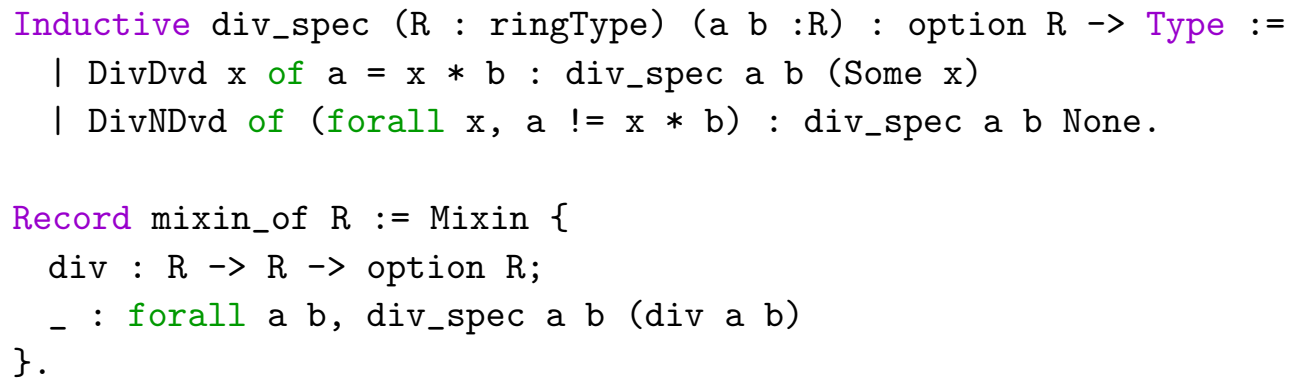

This structure is denoted by DvdRing and for a ring to be an instance it needs to have a function div that returns an option type, such that if div a $\mathrm{b}=$ None then $a \nmid b$, and if $\operatorname{div} \mathrm{a} \mathrm{b}=$ Some $\mathrm{x}$ then $x$ is the witness that $a \mid b$. The notation used for div $\mathrm{a} \mathrm{b}$ in the formalization is a $\% /$ ? b. There is also a $\% /$ notation for the div function that returns a boolean, this relies on a coercion from option to bool defined in the SSREFLECT libraries (mapping None to false and Some $\mathrm{x}$ to true for any $\mathrm{x}$ ). Using this we have implemented the notion of associatedness, denoted by $\%=$, and the basic theory of divisibility.

Next we have the GCDDomain structure which is implemented as:

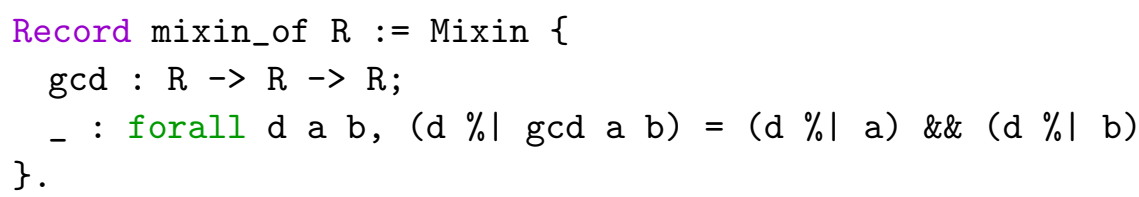

For a ring to be a GCDDomain it needs to have a gcd function satisfying the property above. This property is sufficient as it implicitly gives that $\operatorname{gcd}(a, b) \mid a$ and $\operatorname{gcd}(a, b) \mid b$ since divisibility is reflexive.

The BezoutDomain structure looks like:

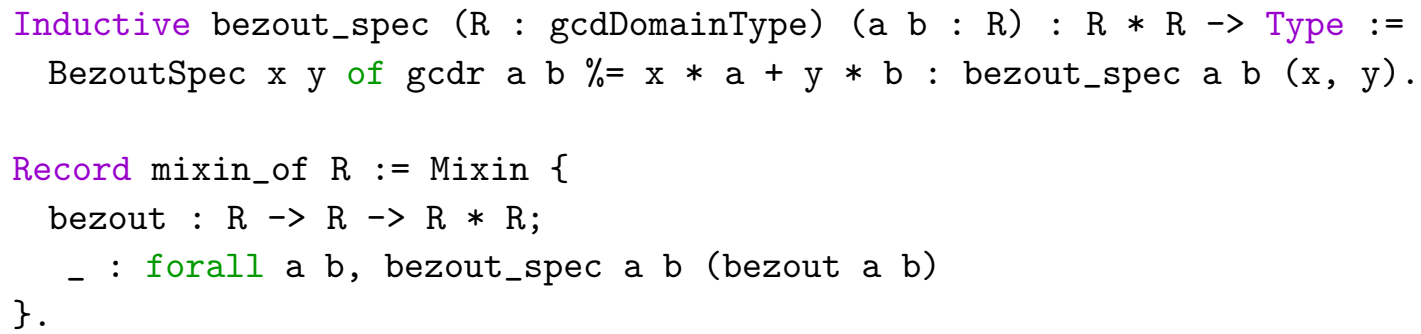


Recall that a constructive principal ideal domain is a Bézout domain where strict divisibility is well-founded. This is denoted by PID and is implemented by:

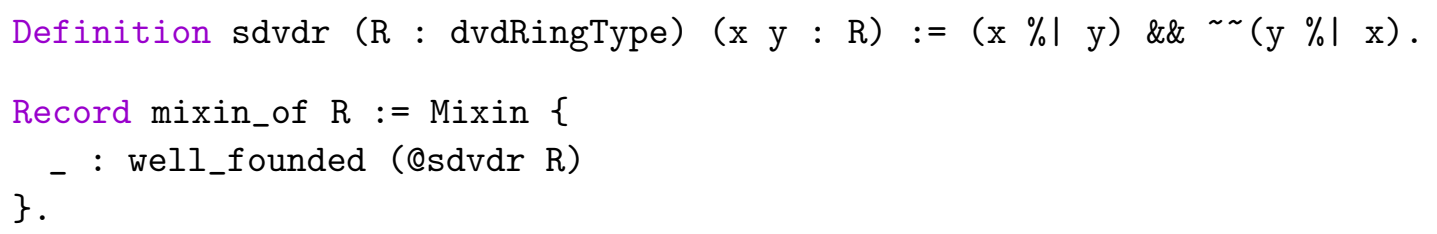

The notation $\mathrm{x} \%<1$ y will be used to denote sdvdr $\mathrm{x}$ y and $\sim$ denotes the boolean negation. We will see more precisely in section 4.2 how well_founded is defined formally in CoQ's standard library when we use it to prove the termination of our Smith normal form algorithm.

We also have the EuclideanDomain structure that represents Euclidean domains:

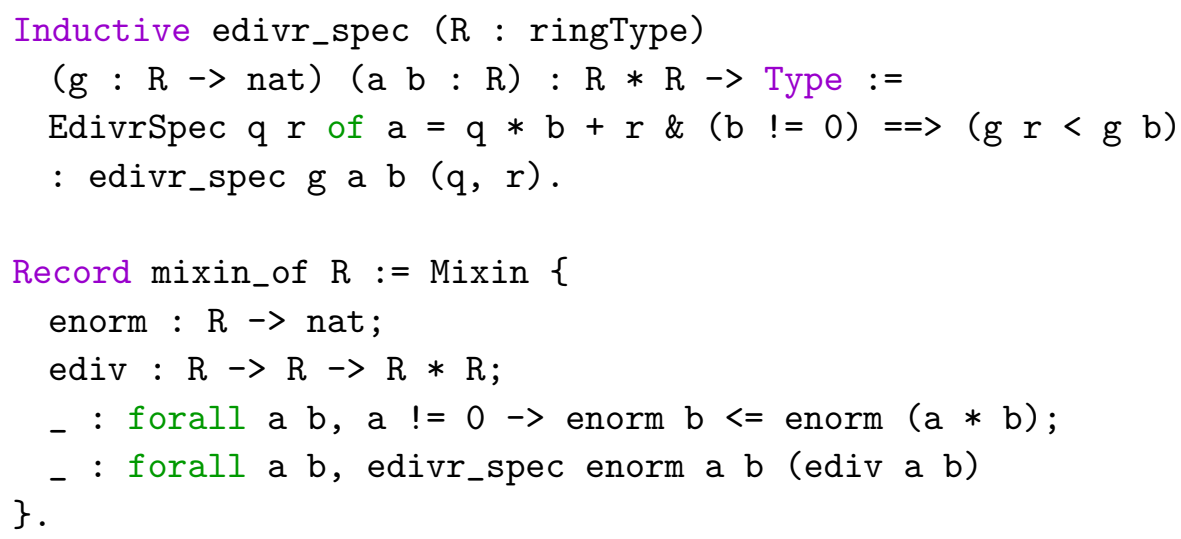

This structure contains the Euclidean norm and the Euclidean division function together with their proofs of correctness. We have implemented the extended version of Euclid's algorithm for Euclidean domains and proved that it satisfies bezout_spec. Hence we get that Euclidean domains are Bézout domains. We have also proved that any EuclideanDomain is a PID which means that strict divisibility is well-founded in both $\mathbb{Z}$ and $k[x]$.

The relationship between the algebraic structures presented in this section can be depicted by:

EuclideanDomain $\subset$ PID $\subset$ BezoutDomain $\subset$ GCDDomain $\subset$ DvdRing $\subset$ IntegralDomain

where IntegralDomain is already present in the SSREFLECT hierarchy. In the next section we consider an algorithm for computing the Smith normal form of matrices over the first two algebraic structures in the chain of inclusions. This means that these two structures are elementary divisor rings. In section 6 we will generalize to Bézout domains of Krull dimension $\leq 1$ and adequate domains that fit in between PID and BezoutDomain in the chain of inclusions.

\section{A verified Algorithm for the Smith Normal Form}

In 25] Kaplansky introduced the notion of elementary divisor rings as rings where every matrix is equivalent to a matrix in Smith normal form, that is, given a $m \times n$ matrix $M$ there exist invertible matrices $P$ and $Q$ of size $m \times m$ and $n \times n$ respectively, such that $P M Q=D$ where $D$ is a diagonal matrix of the form: 


$$
\left[\begin{array}{cccccc}
d_{1} & & 0 & \cdots & \cdots & 0 \\
& \ddots & & & & \vdots \\
0 & & d_{k} & 0 & \cdots & 0 \\
\vdots & & 0 & 0 & & \vdots \\
\vdots & & \vdots & & \ddots & \vdots \\
0 & \cdots & 0 & \cdots & \cdots & 0
\end{array}\right]
$$

with the additional property that $d_{i} \mid d_{i+1}$ for all $i$.

Let us first explain how we formalized the notion of Smith normal form in CoQ, with the following representation of matrices taken from the SSREFLECT library:

Inductive matrix $\mathrm{R} m \mathrm{n}:=$ Matrix of $\{$ ffun 'I_m * 'I_n $\rightarrow \mathrm{R}$ \}.

Here 'I_m is the type of ordinals (i.e. natural numbers bounded by $\mathrm{m}$ ) which has exactly $\mathrm{m}$ inhabitants and can be coerced to nat. Matrices are then implemented as finite functions over finite sets of indices, with dependent types being used to ensure well-formedness. We use the notation ' $M[R]_{-}(m, n)$ for the type matrix $R m n$, the notation ' $r V[R]_{-} m$ for the type of row vectors of length $m$ and the notation ' $c V[R] \_m$ for column vectors of height $m$. The ring $R$ is often omitted from these notations when it can be inferred from the context.

In order to express that a matrix is in Smith normal form, we define diag_mx_seq, which rebuilds a diagonal matrix from a list (note that the type of lists is called seq in the SSREFLECT library) of diagonal coefficients:

Definition diag_mx_seq $\mathrm{m} n$ ( $\mathrm{s}$ : seq $\mathrm{R})$ :=

$\backslash \operatorname{matrix}(i<m, j<n) s^{\prime}{ }_{-} i *+(i==j:>n a t)$.

The notation $\mathrm{x} *+\mathrm{n}$, where $\mathrm{x}$ belongs to a ring and $\mathrm{n}$ is a natural number, stands for the sum $\mathrm{x}+\ldots+\mathrm{x}$ iterated $\mathrm{n}$ times. In the expression of the general coefficients of the matrix above, $i$ and $j$ are ordinals of type 'I_m and 'I_n respectively. The notation $i==j:>$ nat tells CoQ to compare them as natural numbers and returns a boolean. A coercion then sends this boolean to a natural number (true is interpreted by 1 and false by 0 ). Thus $s^{\prime} \_i *+(i==j:>$ nat) denotes the element of index $i$ in $s$ if $i$ and $j$ have the same value, 0 otherwise.

Now if $M$ is a matrix, an algorithm for computing the Smith normal form should return a list $\mathbf{s}$ and two matrices $P$ and $Q$ such that:

- The sequence $\mathbf{s}$ is sorted for the divisibility relation.

- The matrix diag_mx_seq $\mathrm{m} \mathrm{n}$ s is equivalent to $\mathrm{M}$, with transition matrices $\mathrm{P}$ and $\mathrm{Q}$.

Which translates formally to an inductive predicate:

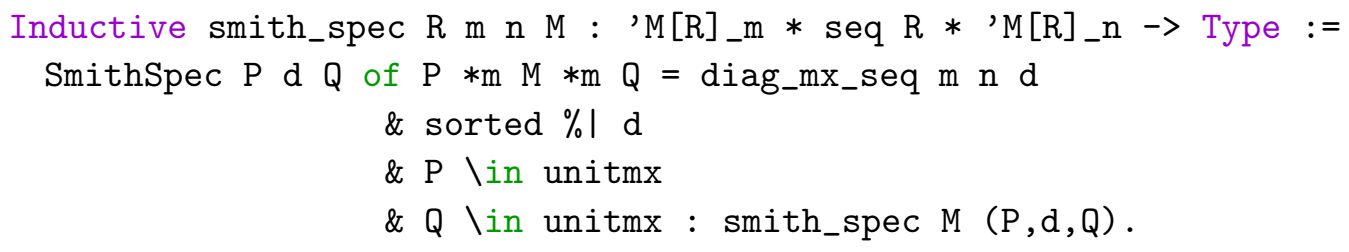

We have packaged this in the same manner as above in order to represent elementary divisor rings: 


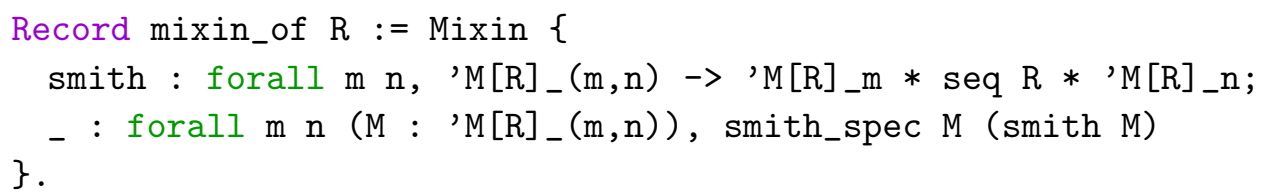

In the rest of this section we will see direct proofs that Euclidean domains and constructive principal ideal domains provide instances of this structure.

4.1. Smith normal form over Euclidean domains. We mentioned in the introduction that constructive finite dimensional linear algebra over a field can be reduced to matrix encodings. Information like the rank and determinant is then reconstructed from the encoding using Gaussian elimination, which involves three kinds of operations on the matrix:

(1) Swapping two rows (resp. columns)

(2) Multiplying one row (resp. column) by a nonzero constant

(3) Adding to a row (resp. column) the product of another one by a constant

These three operations are interesting because they are compatible with matrix equivalence. In particular, they can be expressed as left (resp. right) multiplication by invertible matrices.

The same algorithm fails to apply in general to a matrix over a ring, since it may require a division by the pivot, which could be not exact. The content of this section can thus be seen as a generalization of Gaussian elimination to Euclidean domains.

To make this extension possible, a new kind of elementary operations needs to be introduced. Let $a$ and $b$ be elements of a Euclidean domain $R$. Bézout's identity gives $u$ and $v$ such that $u a+v b=\gamma$ where $\gamma=\operatorname{gcd}(a, b)$. Let us note $a^{\prime}=\frac{a}{\gamma}$ and $b^{\prime}=\frac{b}{\gamma}$, these divisions being exact by definition of the gcd. We get the identity: $u a^{\prime}+v b^{\prime}=1$. Consider the following square matrix of size $n$ :

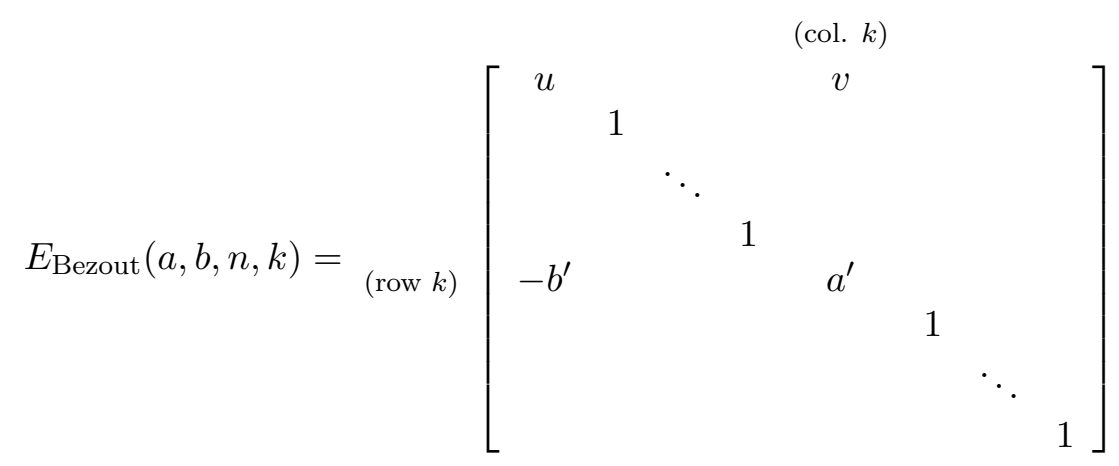

The coefficients not explicitly shown in this matrix are assumed to be zeros. Note that $\operatorname{det}\left(E_{\text {Bezout }}(a, b, n, k)\right)=u a^{\prime}+v b^{\prime}=1$, so in particular the matrix above is invertible.

We formalize these matrices as follows:

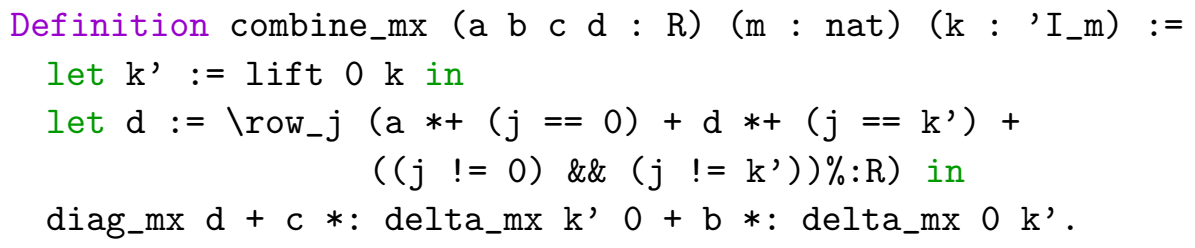


Definition Bezout_mx (a b : R) (m : nat) (k : 'I_m) := let: $\left(\_, \mathrm{u}, \mathrm{v}, \mathrm{a} 1, \mathrm{~b} 1\right):=\operatorname{egcdr} \mathrm{a}$ b in combine_mx u v (-b1) a1 k.

For an ordinal $i$ of type 'I_m, lift 0 i represents the ordinal $1+i$ of type ' $I_{-}(1+m)$. The notation \row_ $(j<m) r j$ corresponds to the row matrix $[r 0, \ldots, r(m-1)]$, if the dimension can be automatically inferred then we can just write $\backslash r_{0} w_{-} j \quad j$. If $b$ is a boolean, the term $b \%: R$ reduces to 1 if $b$ is true, 0 otherwise. The matrix diag_mx $d$ correspond to the diagonal matrix where diagonal coefficients are the coefficients of the row matrix $\mathrm{d}$, and delta_mx $i \mathrm{j}$ is the matrix which has only zeros except at position $(i, j)$, where the coefficient is 1 . Finally, a $*$ : A is the matrix A multiplied by the scalar a. Note that the Bézout identity between $a$ and $b$ is given by the function egcdr, which is exported by the underlying Euclidean ring.

Like other elementary operations, multiplication by $E_{\text {Bezout }}(a, b, n, k)$ on the left corresponds to an operation on the rows:

$$
E_{\text {Bezout }}(a, b, n, k) \times\left[\begin{array}{c}
L_{1} \\
L_{2} \\
\vdots \\
L_{k-1} \\
L_{k} \\
L_{k+1} \\
\vdots \\
L_{n}
\end{array}\right]=\left[\begin{array}{c}
u L_{1}+v L_{k} \\
L_{2} \\
\vdots \\
L_{k-1} \\
-b^{\prime} L_{1}+a^{\prime} L_{k} \\
L_{k+1} \\
\vdots \\
L_{n}
\end{array}\right]
$$

These row operations are described formally by:

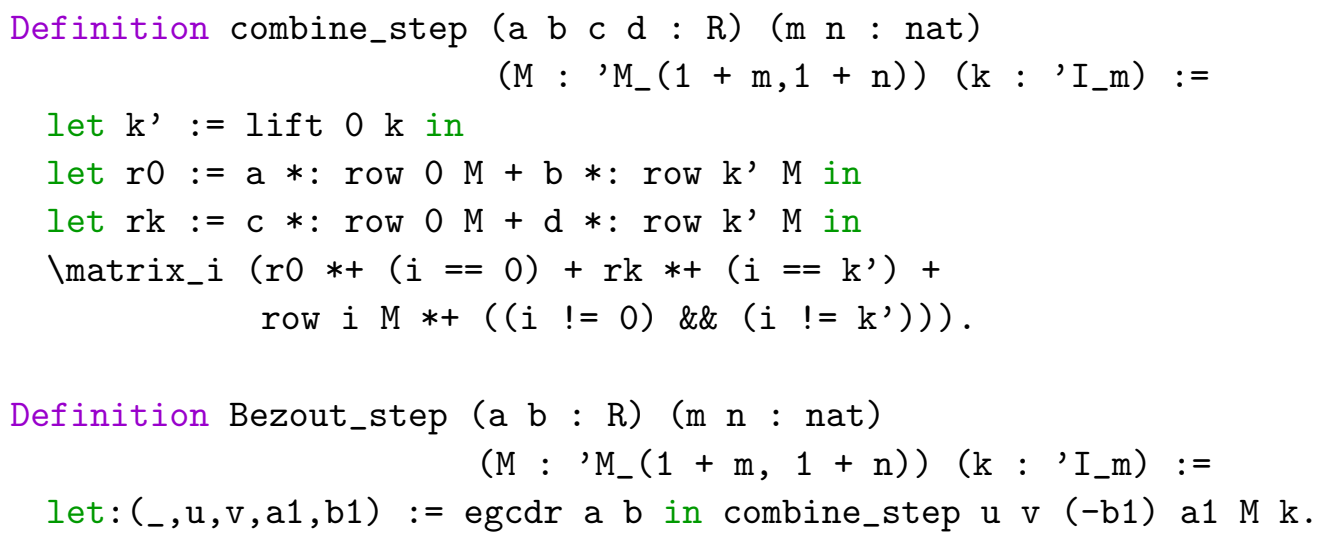

Here row $\mathrm{i}$ M represents the $i$ :th row of M. A lemma connects these row operations to the corresponding elementary matrices:

Lemma Bezout_stepE a b (m n : nat) (M : ' $\left.M_{-}(1+m, 1+n)\right) k$ :

Bezout_step a b M k = Bezout_mx a b k $* m$ M.

Let now $M=\left(a_{i, j}\right)$ be a matrix with coefficients in $R$. We will now show how to reduce $M$ to its Smith normal form using elementary operations. As for Gaussian elimination, we start by finding a nonzero pivot $g$ in $M$, which is moved to the upper-left corner (if $M=0, M$ is in Smith normal form). We search the first column for an element which is not divisible by $g$. 
Let us assume that $g \nmid a_{k, 1}$, we then multiply the matrix on the left by $E_{\text {Bezout }}\left(g, a_{k, 1}, n, k\right)$ :

$$
E_{\text {Bezout }}\left(g, a_{k, 1}, n, k\right) \times\left[\begin{array}{cc}
g & L_{1} \\
a_{2,1} & L_{2} \\
\vdots & \vdots \\
a_{k, 1} & L_{k} \\
\vdots & \vdots \\
a_{n, 1} & L_{n}
\end{array}\right]=\left[\begin{array}{cc}
\gamma & u L_{1}+v L_{k} \\
a_{2,1} & L_{2} \\
\vdots & \\
-g^{\prime} g+a^{\prime} a_{k, 1} & -g^{\prime} L_{1}+a^{\prime} L_{k} \\
\vdots & \vdots \\
a_{n, 1} & L_{n}
\end{array}\right]
$$

with the Bézout identity $u g+v a_{k, 1}=\gamma=\operatorname{gcd}\left(g, a_{k, 1}\right)$ and posing as previously $g^{\prime}=\frac{g}{\gamma}$, we have $a^{\prime}=\frac{a_{k, 1}}{\gamma}$.

By definition of $\gamma$, we have: $\gamma \mid-g^{\prime} g+a^{\prime} a_{k, 1}$. Moreover, all the coefficients in the first column of $M$ which were divisible by $g$ are also by $\gamma$. We can therefore repeat this process until we get a matrix whose upper-left coefficient (which we still name $g$ ) divides all the coefficients in the first column. Linear combinations on rows can thence lead to a matrix $B$ of the following shape:

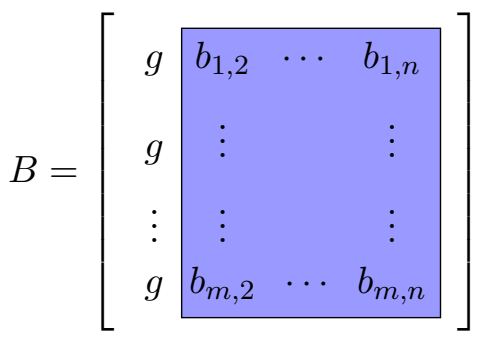

We then search the indicated submatrix of $B$ for an element that is not divisible by $g$. If such a coefficient $b_{i, j}$ is found, it is moved to the top by permuting rows 1 and $i$. Thus $g$ is still the upper-left coefficient ${ }^{2}$ and multiplications on the right by $E_{\text {Bezout }}$ matrices allow, like previously, to obtain a matrix whose upper-left coefficient divides all the others.

This first step is implemented by the function improve_pivot_rec:

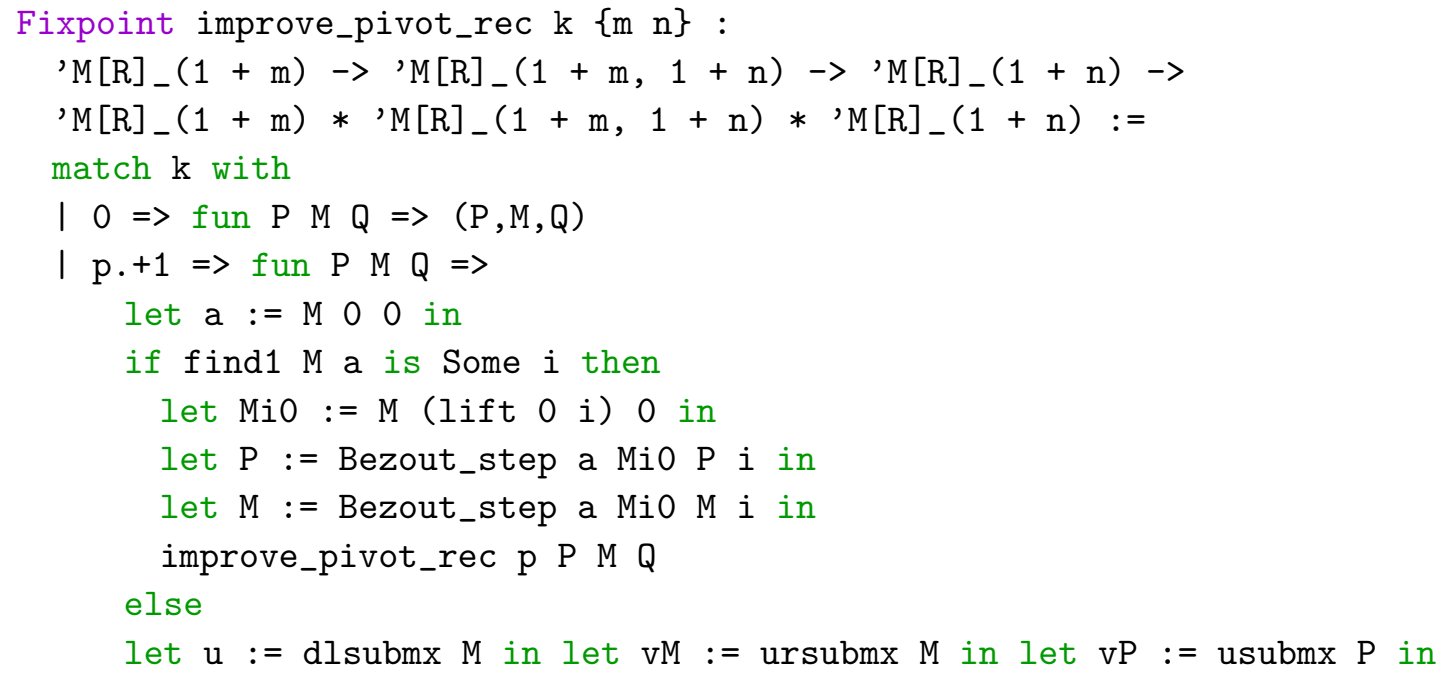

${ }^{2}$ The authors were inspired by the use of a similar trick in an algorithm formalized by Georges Gonthier. 


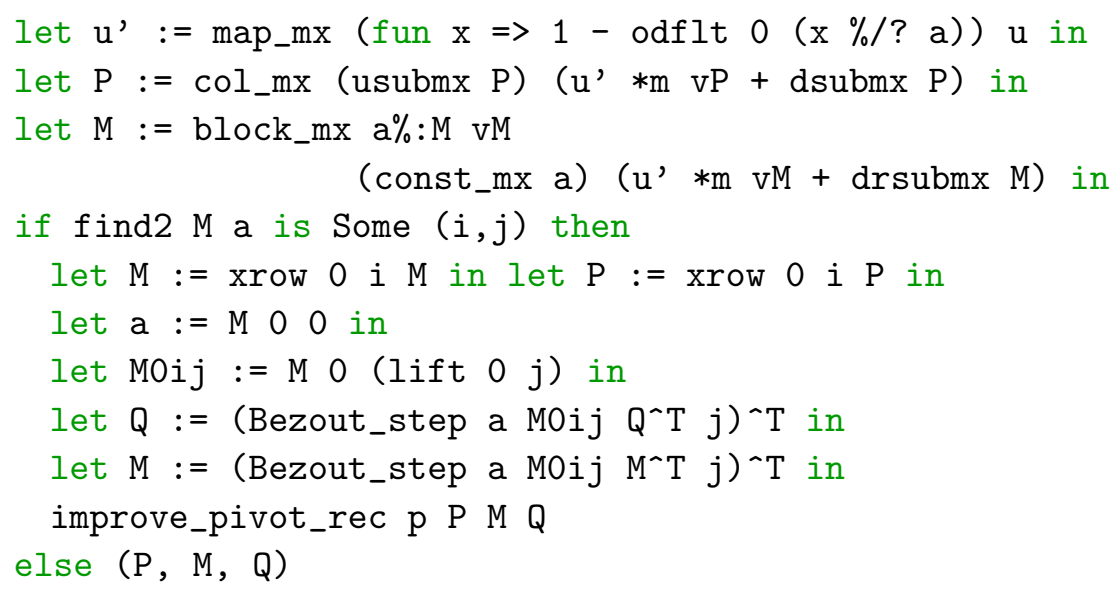

If $A, B, C$ and $D$ are four matrices (with matching dimensions) then block_mx A B C D is the matrix:

$$
M=\left[\begin{array}{ll}
A & B \\
C & D
\end{array}\right]
$$

where $A=$ ulsubmx $M, B=\operatorname{ursubmx} M, C=d l \operatorname{submx} M$ and $D=d r s u b m x ~ M$. Similarly $\mathrm{C}=\mathrm{col}_{-} \mathrm{mx} \mathrm{A} B$ is a column matrix with $\mathrm{A}=$ usubmx $\mathrm{C}$ and $\mathrm{B}=\operatorname{dsubmx} \mathrm{C}$ (the functions for constructing and destructing row matrices have similar names). The matrix const_mx a is the matrix where each coefficient is equal to a and xrow $i j M$ is the matrix $M$ with the rows $i$ and $j$ exchanged.

The function improve_pivot_rec takes as arguments a natural number $\mathrm{k}$ which represents the number of remaining steps, the original matrix and two current transition matrices. If the number of remaining steps is zero, the matrices are returned unchanged (line 5). If not, the first column is searched for an element that is not divisible by the pivot (function find1, line 8). If such an element is found on a row of index $i$, a Bézout step is performed between the first row and the one of index $i$, and the function is called recursively (lines 9 to 12). If, on the contrary, the pivot divides all the elements in the first column, some linear combinations (lines 14 to 18) bring us back to a matrix of the shape of the matrix $B$ seen above. Finally, the remaining lines search the whole matrix for an element that is not divisible by the pivot (function find2), perform a Bézout step on the columns if appropriate, and call the function recursively.

We have made several choices when implementing this function. First, the argument $\mathrm{k}$ bounding the number of steps makes it easy to have a structural recursion (this natural number decreases by 1 at each step). In this usual technique, $\mathrm{k}$ is often called the fuel of the recursion. The flip side is that in order to call the function, an a priori bound on the number of steps has to be provided. It is at this point that the hypothesis we made that $R$ is a Euclidean domain comes in handy: we can take as a bound the Euclidean norm of the upper-left coefficient of the original matrix.

We also chose to abstract over initial transition matrices, which are updated as the process goes on. From a computational standpoint, this approach has two benefits. First, it avoids the need for products by transition matrices, asymptotically more costly than to perform the elementary operations directly. Then, it makes the function improve_pivot_rec tail-recursive, which can have a good impact on performance. 
The flip side is that it is slightly more difficult to express and manipulate formally the link between the matrices taken as arguments and those returned by the function. Indeed, the specification of this function involves inverses of transition matrices:

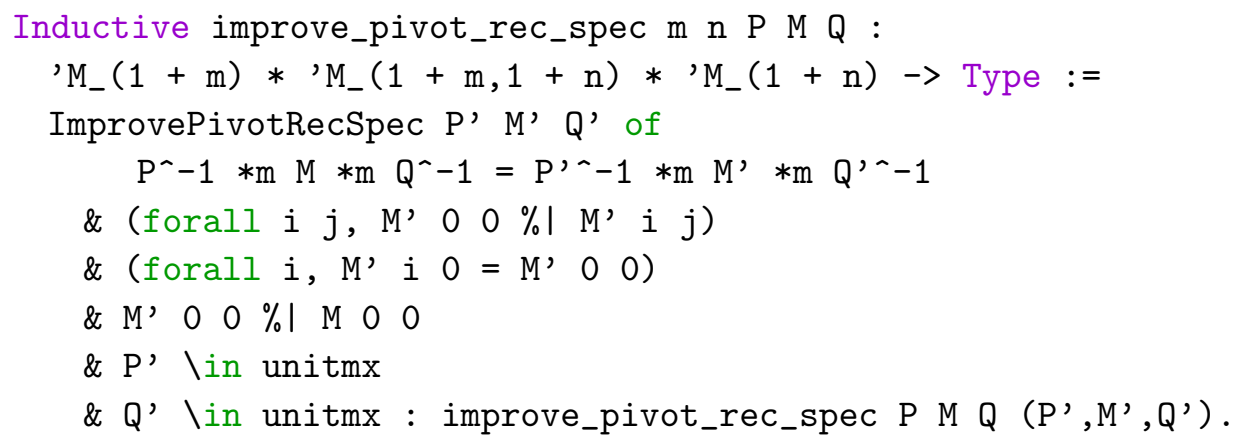

The statement above can be read as follows: given three matrices $P, M$ and $Q$, a triple $\left(P, M^{\prime}, Q^{\prime}\right)$ satisfies the specification if applying to $M$ the inverse of elementary operations represented by the initial transition matrices $P$ and $Q$ gives the same result as applying the inverses of the transition matrices $\mathrm{P}^{\prime}$ and $\mathrm{Q}^{\prime}$ to $\mathrm{M}^{\prime}$.

The correctness lemma of the function improve_pivot_rec states that for an initial matrix $M$ whose upper-left coefficient is nonzero and has a norm smaller than a natural number $\mathrm{k}$, and for invertible matrices $\mathrm{P}$ and $\mathrm{Q}$, the triple returned by improve_pivot_rec k P M Q satisfies the specification represented by the inductive type improve_pivot_rec_spec:

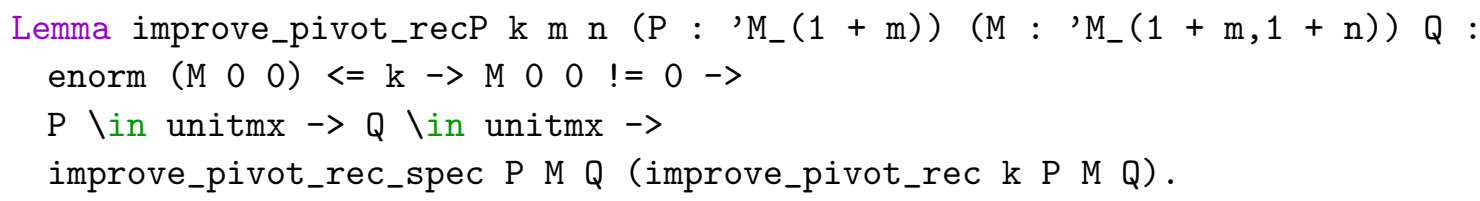

Initially, we call the function improve_pivot_rec with identity transition matrices:

Definition improve_pivot $\mathrm{k} \mathrm{m} \mathrm{n}\left(\mathrm{M}:{ }^{\prime} \mathrm{M}_{-}(1+\mathrm{m}, 1+\mathrm{n})\right):=$ improve_pivot_rec $\mathrm{k} 1 \%: \mathrm{M}$ M $1 \%: \mathrm{M}$.

By successive subtractions of the first row from all the others and then by linear combinations of columns, we get a matrix $C$ :

where $g$ divides all coefficients of $C^{\prime}$.

$$
C=\left[\begin{array}{c|ccc}
g & 0 & \cdots & 0 \\
\hline 0 & & & \\
\vdots & & & C^{\prime} \\
0 & & &
\end{array}\right]
$$

The global algorithm computing the Smith normal form proceeds as follows: it stores the pivot $g$ obtained after the previous step, then divides all coeficients of $C^{\prime}$ by $g$ and is applied recursively to the resulting matrix. Let us pose $k=\min (m, n)$. From the pivots $g_{1}, \ldots, g_{k}$ obtained, the final output of the algorithm is given by the following sequence $d_{1}, \ldots, d_{k}$ :

$$
d_{1}, d_{2}, \ldots, d_{k}=g_{1}, g_{1} g_{2}, \ldots, \prod_{i=1}^{k} g_{i}
$$


The Smith normal form of the original matrix is then the following diagonal matrix of size $m \times n$ :

$$
\left[\begin{array}{ccccccc}
d_{1} & & & & & & \\
& d_{2} & & & & & \\
& & \ddots & & & & \\
& & & d_{k} & & & \\
& & & & 0 & & \\
& & & & & \ddots & \\
& & & & & & 0
\end{array}\right]
$$

This global procedure is implemented by the function Smith :

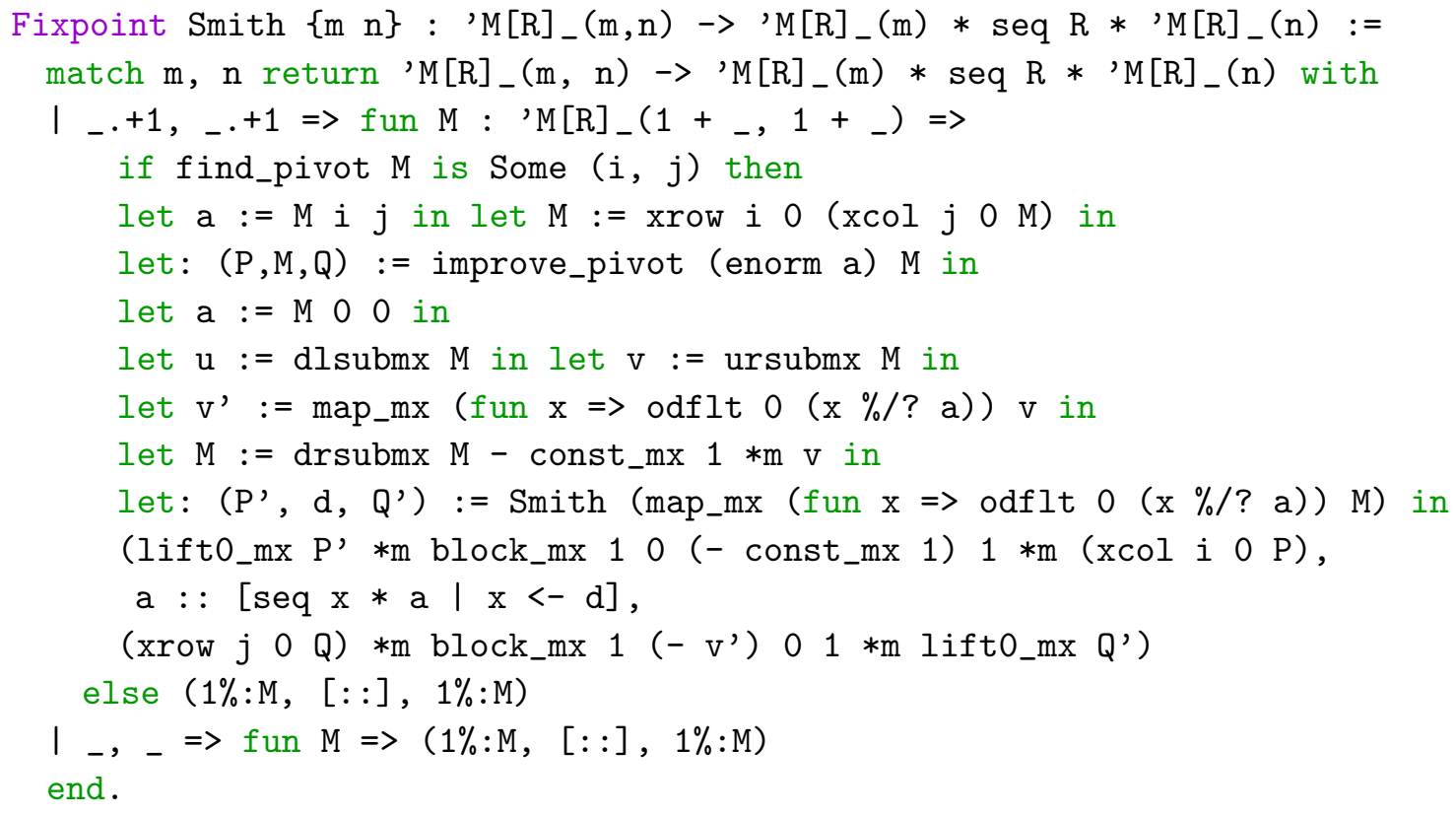

If $M$ has type 'M[R]_n then lift0_mx $M=$ block_mx 1000 of type 'M[R]_(1 + n). The notation [seq $f \mathrm{x} \mid \mathrm{x}<-\mathrm{xs}$ ] is like a list comprehension in HASKELL and means map f xs.

The function Smith takes as argument a matrix and returns a sequence made of the nonzero diagonal coefficients of its Smith form, as well as the corresponding transition matrices. The first step (lines 4 and 5) consists in searching for a nonzero pivot in the whole matrix and moving it in the upper-left position. If no pivot is found, all the coefficients are zero and an empty sequence is therefore returned. Otherwise, the function improve_pivot defined previously is called (line 6), then some elementary row operations are performed (lines 8 to 10) to get a matrix of the shape of the matrix $C$ shown above. The bottom-right submatrix is then divided by the pivot and a recursive call is performed (line 11). The sequence of coefficients and transition matrices obtained are then updated (lines 12 to 14).

We have stated and proved the following correctness lemma:

Lemma SmithP (m $n$ : nat) (M : 'M_ $(m, n))$ : smith_spec M (Smith M).

Using this we have instantiated the structure of elementary divisor rings on Euclidean domains. 
4.2. Extension to principal ideal domains. We mentioned in section 3 that constructive principal ideal domains were Bézout domains with a well-founded divisibility relation. Wellfoundedness is defined in COQ's standard library using an accessibility predicate [31]:

Inductive Acc (A : Type) ( $\mathrm{R}: \mathrm{A} \rightarrow \mathrm{A} \rightarrow$ Prop) (x : A) : Prop := Acc_intro : (forall y : A, R y $x \rightarrow A c c R y) \rightarrow A c c R x$.

The idea is that all objects of the inductive type Acc have to be built by a finite number of applications of the constructor Acc_intro. Hence, for any a such that Acc R a, all chains $\left(\mathrm{x}_{n}\right)$ such that $\mathrm{R} \mathrm{x}_{n+1} \mathrm{x}_{n}$ and $\mathrm{x}_{0}=\mathrm{a}$ have to be finite. Note however that there can be infinitely many elements $\mathrm{x}$ such that $\mathrm{R} \mathrm{x}$ a. Using this definition of accessibility, we can now state that a relation over a type $\mathrm{A}$ is well-founded if all elements in $\mathrm{A}$ are accessible:

Definition well_founded (A : Type) ( $\mathrm{R}$ : $\mathrm{A} \rightarrow \mathrm{A} \rightarrow$ Prop) :=

forall a, Acc $\mathrm{R}$ a.

Remember that in the previous section, we used the hypothesis that the ring of coefficients was Euclidean when we computed an a priori bound on the number of steps the function improve_pivot needed to perform. To extend the algorithm to principal ideal domains, we replace the recursion on this bound with a well-founded induction on the divisibility relation.

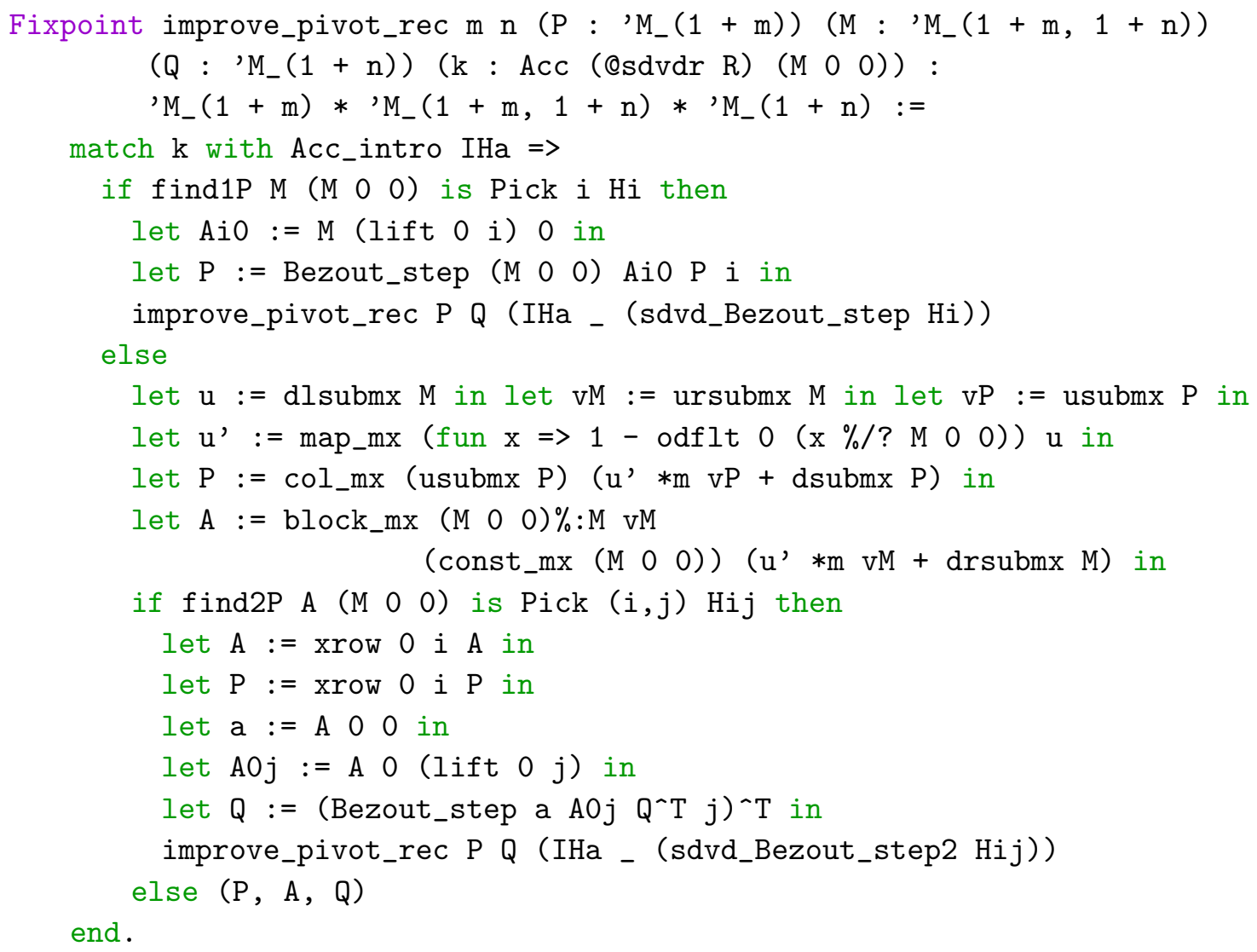

The main difference with the function improve_pivot defined in section 4.1 is that we need to prove that the upper-left element of the matrix on which we make the recursive call is strictly smaller than the one of the original matrix. To build these proofs, we use the functions find1P and find2P which have more expressive (dependent) types than their 
counterparts find1 and find2 that we used previously. They return not only an element of the matrix given as argument, but also a proof that the pivot does not divide this element.

This proof is then used to show that the upper-left coefficient of the matrix decreases, thanks to the following two lemmas:

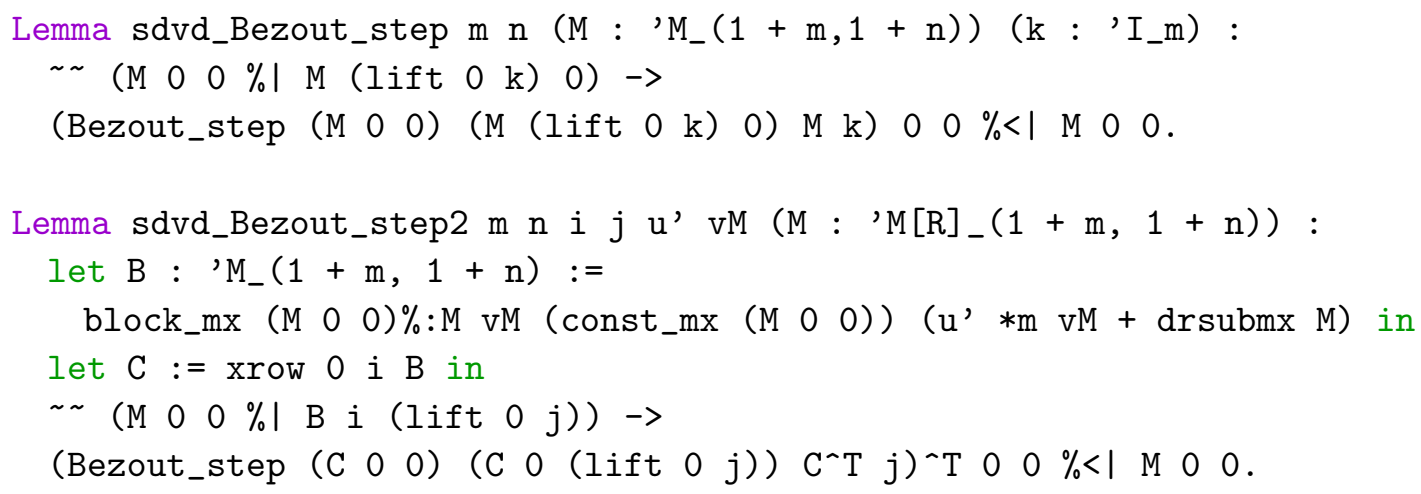

Now, to define the improve_pivot function, we use the hypothesis sdvdr_wf that the divisibility relation is well-founded:

Definition improve_pivot $m \mathrm{n}\left(\mathrm{M}:{ }^{\prime} \mathrm{M}_{-}(1+\mathrm{m}, 1+\mathrm{n})\right):=$ improve_pivot_rec 11 (sdvdr_wf (M 000$)$ ).

The function Smith of section 4.1 is essentially unchanged, the only difference being that we removed the first argument of improve_pivot (which was an a priori bound on the number of steps of improve_pivot_rec).

We have shown how to compute the Smith normal form on Euclidean domains and more generally on principal ideal domains. In the next section, we will explain how to develop a constructive theory of linear algebra based on the existence of such an algorithm.

\section{ELEMENTARY DIVISOR RINGS}

The goal of this section is to develop some theory about linear algebra over elementary divisor rings and discuss the formalization of the classification theorem for finitely presented modules over these rings.

5.1. Linear algebra over elementary divisor rings. One of the key operations in linear algebra is to compute solutions to systems of equations. A suitable algebraic setting for doing so is rings where every finitely generated ideal is finitely presented. These rings are called coherent:

Definition 5.1. A ring is coherent if for any matrix $M$ it is possible to compute a matrix $L$ such that:

$$
X M=0 \leftrightarrow \exists Y \cdot X=Y L
$$

This means that $L$ generates the module of solutions of $X M=0$, i.e. that $L$ generates the kernel of $M$. The notion of coherent rings is usually not mentioned in classical presentations of algebra since Noetherian rings are automatically coherent, but in a computationally meaningless way. It is however a fundamental notion, both conceptually [26, 30] and computationally [1, 2]. Coherent rings have previously been represented in CoQ [9] so we 
will not discuss the details of the formalization here. Instead we show that elementary divisor rings are coherent.

Let $M$ be a $m \times n$ matrix with coefficients in an elementary divisor ring. There are invertible matrices $P$ and $Q$ such that $P M Q=D$ where $D$ is a diagonal matrix in Smith normal form. The rank of $M$, denoted $r(M)$, is the number of nonzero elements of $D$. The kernel of $M$ can be computed by:

$$
\operatorname{ker}(M)=\left(I_{m}-I_{r(M)}\right) P
$$

where $I_{m}$ is a $m \times m$ identity matrix and $I_{r(m)}$ is a $m \times m$ partial identity matrix with $r(M)$ ones on the diagonal and then zeros. The idea behind this definition is that:

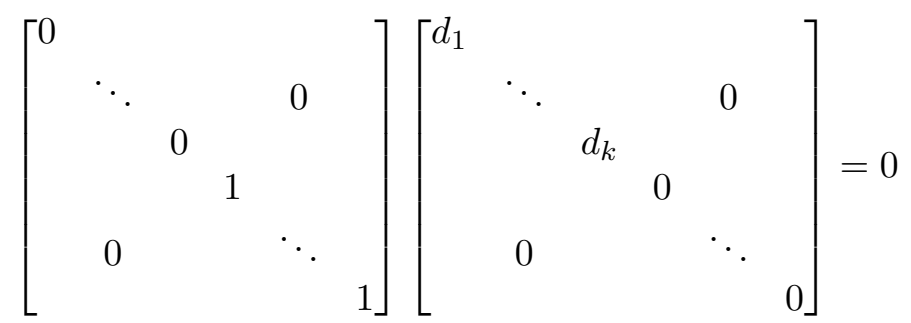

So $\operatorname{ker}(M) M Q=0$ and since $Q$ is invertible, we have $\operatorname{ker}(M) M=0$. We can implement the rank operator and state its correctness by:

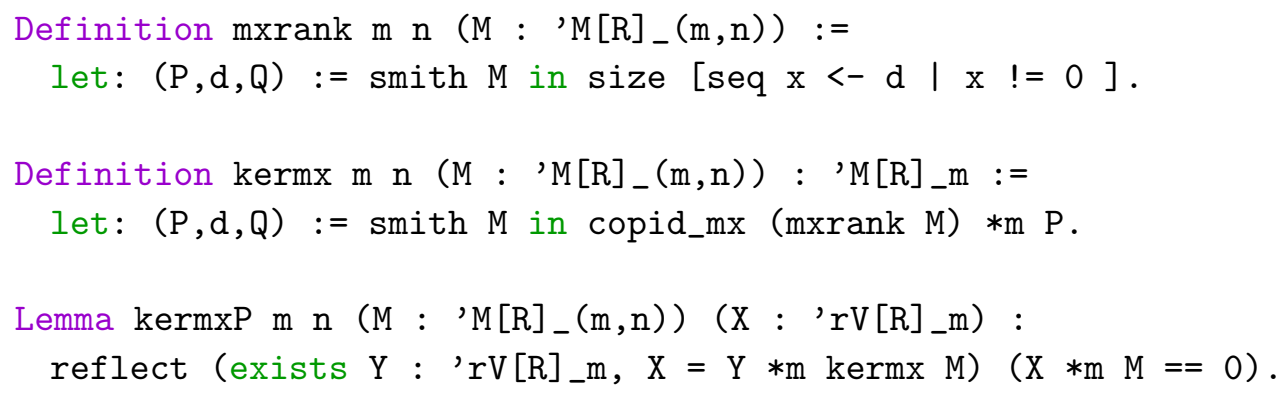

where copid_mx corresponds to the partial identity matrix. The reflect statement should be read as: the boolean equality $\mathrm{X} * \mathrm{~m} \mathrm{M}==0$ holds if and only if there exists $\mathrm{Y}$ : ' $r \mathrm{~V}[\mathrm{R}] \_\mathrm{m}$ such that $\mathrm{X}=\mathrm{Y} * \mathrm{~m}$ kermx $\mathrm{M}$.

An algorithm computing the cokernel of a matrix can be implemented in a similar fashion. This way we have implemented a small library inspired by the one on matrix algebra for fields of SSREFLECT [17, but based on Smith normal form instead of Gaussian elimination.

Another important notion in constructive algebra is strongly discrete rings:

Definition 5.2. A ring is strongly discrete if membership in finitely generated ideals is decidable and if whenever $x \in\left(x_{1}, \ldots, x_{n}\right)$, there exists $y_{1}, \ldots, y_{n}$ such that $x=\sum_{i} x_{i} y_{i}$.

If a ring is both coherent and strongly discrete it is not only possible to solve homogeneous systems of equations but also arbitrary systems of the kind $X M=B$ where $X$ is a $m \times n$ matrix, $M$ a $n \times k$ matrix and $B$ a nonzero $m \times k$ matrix.

It is easy to see that Bézout domains are strongly discrete as any finitely generated ideal is principal. To test if $x \in\left(a_{1}, \ldots, a_{n}\right)$ first compute a principal ideal $(g)$ equivalent to $\left(a_{1}, \ldots, a_{n}\right)$ and then test if $g \mid x$. If this is the case we may construct the witness and otherwise we know that $x \notin\left(a_{1}, \ldots, a_{n}\right)$. 
It is also straightforward to prove that any elementary divisor ring is a Bézout domain. Given $a, b \in R$ we can compute the Smith normal form of a row matrix containing $a$ and $b$. This gives us an invertible $1 \times 1$ matrix $P$, an invertible $2 \times 2$ matrix $Q$, and $g \in R$ such that:

$$
P\left[\begin{array}{ll}
a & b
\end{array}\right] Q=\left[\begin{array}{ll}
g & 0
\end{array}\right]
$$

As $P$ and $Q$ are invertible we get that $g$ is the greatest common divisor of $a$ and $b$. The Bézout coefficients are then found by performing the matrix multiplications on the left-hand side of the equality. Hence we get that elementary divisor rings are not only coherent but also strongly discrete.

In section 6 we consider extensions to Bézout domains that make them elementary divisor rings and hence form a good setting for doing linear algebra. The next subsection shows that the existence of an algorithm for computing the Smith normal form makes finitely presented modules over elementary divisor rings especially well-behaved.

5.2. Finitely presented modules over elementary divisor rings. Recall that a module is said to be finitely presented if it can be described using a finite set of generators and a finite set of relations among these. A convenient way to express this is:

Definition 5.3. An $R$-module $\mathcal{M}$ is finitely presented if there is an exact sequence:

$$
R^{m_{1}} \stackrel{M}{\longrightarrow} R^{m_{0}} \stackrel{\pi}{\longrightarrow} \mathcal{M} \longrightarrow 0
$$

This means that $\pi$ is a surjection and $M$ a matrix representing the $m_{1}$ relations among the $m_{0}$ generators of the module $\mathcal{M}$. Another way to think of $\mathcal{M}$ is as the cokernel of $M$, that is, $\mathcal{M} \simeq \operatorname{coker}(M)=R^{m_{0}} / \mathcal{I m}(M)$. So a module has a finite presentation if it can be expressed as the cokernel of a matrix. As all information of finitely presented modules is contained in its presentation matrix we get that all algorithms on finitely presented modules can be described by manipulating the presentation matrices [11, 19, 26].

A morphism $\varphi$ between finitely presented modules $\mathcal{M}$ and $\mathcal{N}$ given by presentations:

$$
R^{m_{1}} \stackrel{M}{\longrightarrow} R^{m_{0}} \longrightarrow \mathcal{M} \longrightarrow 0 \quad R^{n_{1}} \stackrel{N}{\longrightarrow} R^{n_{0}} \longrightarrow \mathcal{N} \longrightarrow 0
$$

is represented by a $m_{0} \times n_{0}$ matrix $\varphi_{G}$ and a $m_{1} \times n_{1}$ matrix $\varphi_{R}$ such that the following diagram commutes:

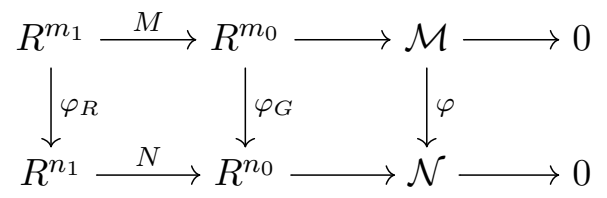

The intuition why two matrices are needed is that the morphism affects both the generators and relations of the modules, hence the names $\varphi_{G}$ and $\varphi_{R}$. In this paper we adopt the SSREFLECT convention that composition is read in diagrammatic order (i.e. from left to right) when writing equations obtained from commutative diagrams. This means that the equation related to the above diagram is written $M \varphi_{G}=\varphi_{R} N$.

In order for us to be able to compute kernels of morphisms we need to assume that the underlying ring is coherent so that we can solve systems of equations involving the underlying matrices. If the underlying ring is also strongly discrete, it is possible to represent morphisms using only $\varphi_{G}$ and a proof that $\exists X . X N=M \varphi_{G}$ as any system of equations of the kind $X M=B$ is solvable. Two of the authors have previously [7] formalized finitely 
presented modules over coherent and strongly discrete rings in COQ which provides a basis for this part of the formalization.

It is in general not possible to decide if two finitely presented modules are isomorphic or not. However, if the underlying ring is an elementary divisor ring, it becomes possible. Indeed, let $R$ be an elementary divisor ring and $M$ be a $m_{1} \times m_{0}$ matrix presenting an $R$-module $\mathcal{M}$. As $M$ is equivalent to a diagonal matrix $D$, there are invertible matrices $P$ and $Q$ such that $M Q=P^{-1} D$. This gives a commutative diagram:

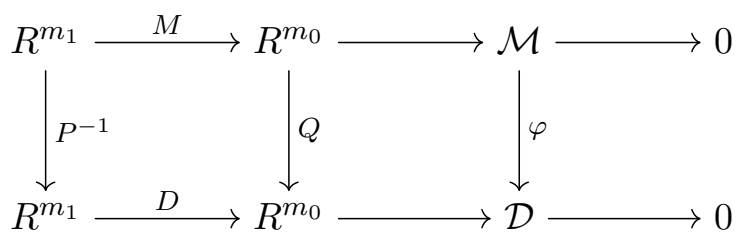

We can further prove that $\varphi$ is an isomorphism as $P$ and $Q$ are invertible, and hence get that $\mathcal{M} \simeq \mathcal{D} \simeq \operatorname{coker}(D)$. Now, since $D$ is a diagonal matrix with nonzero elements $d_{1}, \ldots, d_{n} \in R$ on the diagonal, we get that:

$$
\mathcal{M} \simeq R^{m_{0}-n} \oplus R /\left(d_{1}\right) \oplus \cdots \oplus R /\left(d_{n}\right)
$$

with the additional property that $d_{i} \mid d_{i+1}$ for all $1 \leqslant i<n$. Note that if $d_{i}$ is a unit then $R /\left(d_{i}\right) \simeq 0$. This means that the theory of finitely presented modules over elementary divisor rings $R$ is particularly well-behaved as any finitely presented $R$-module $\mathcal{M}$ can be decomposed into a direct sum of a free module and cyclic modules. This is the first part of the classification theorem for finitely presented modules over elementary divisor rings, the second part is the fact that the $d_{i}$ are unique up to multiplication by units which makes the decomposition unique.

The uniqueness part is also necessary in order to get a decision procedure for the isomorphism of finitely presented modules over elementary divisor rings. So far we only know that any module may be decomposed as above, but there is, a priori, no reason why two isomorphic modules should have related decompositions.

In the next section we will see that the Smith normal form is unique up to multiplication by units if the underlying ring has a gcd operation, which in turn completes the classification theorem and gives us a decision procedure for module isomorphism.

5.3. Uniqueness of the Smith normal form. The formal proof that the Smith normal form is unique up to multiplication by units presented here is based on [4]. In order to formalize this proof we need to represent minors (determinants of submatrices) in CoQ. This notion was defined in a previous work on formalizing the Sasaki-Murao algorithm computing the characteristic polynomial of a matrix [10. With the SSREFLECT definition of matrices it is easy to give a definition of submatrices (denoted by $M(f, g)$ ) and minors:

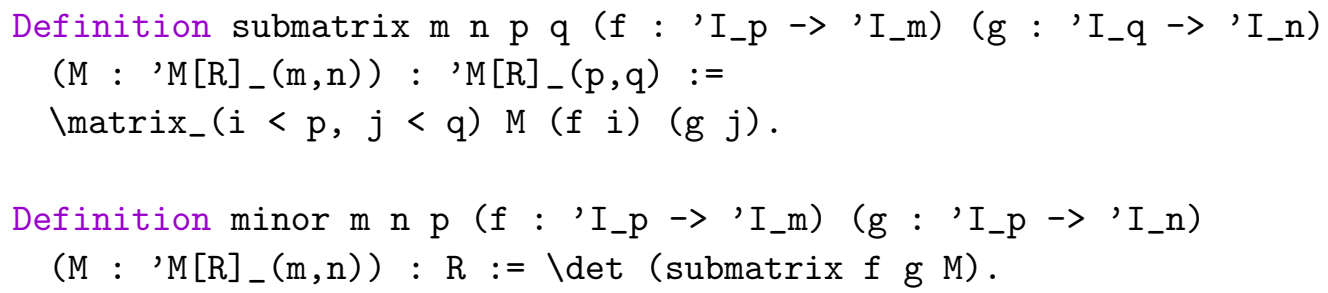


For example, the rows (resp. columns) of the matrix $M(f, g)$ are the rows (resp. columns) $f(0), f(1), \ldots$ (resp. $g(0), g(1), \ldots)$ of $M$. It would be natural to define submatrices only when $f$ and $g$ are strictly increasing, however this is not necessary as many theorems are true for arbitrary functions. We denote $\mathrm{p}$ in the definition of minor above as the order of the minor, that is, a minor of order $p$ is the determinant of a submatrix of dimension $p \times p$.

The key result in order to prove the uniqueness theorem for the Smith normal form is that the product of the $k$ first elements of the diagonal in the Smith normal form is associated to the gcd of the minors of order $k$ of the original matrix. More precisely, let $M$ be the original matrix and $d_{i}$ the $i$ :th element of the diagonal in the Smith normal form of $M$, also let $\vec{m}_{k}$ be the minors of order $k$ of $M$, then the statement is:

$$
\prod_{i=1}^{k} d_{i}=\operatorname{gcd}\left(\vec{m}_{k}\right)
$$

Using the big operators library of SSREFLECT [3] this can be expressed compactly as:

Lemma Smith_gcdr_spec :

$\backslash$ prod_(i<k) d'_i \%= \big[gcdr/0]_f \big[gcdr/0]_g minor f g M.

The order of the minors that we consider are given by the types of $f$ and $g$. For the sake of readability, we have omitted these types.

The first step in proving this is by showing that it holds for the Smith normal form of $M$, namely the diagonal matrix $D$. Since it is a diagonal matrix, the only nonzero minors of order $k$ are the determinants of diagonal matrices of dimension $k \times k$, that are products of $k$ elements of the diagonal of $D$. Also, since each element of the diagonal divides the next one, the greatest common divisor of the minors of order $k$ is the product of the $k$ first elements of the diagonal. For example, if the diagonal is $(a, b, c)$ with $a \mid b$ and $b \mid c$ then $\operatorname{gcd}(a b, b c, a c)=a b$.

The next step is to prove that the gcd of the minors of order $k$ of $M$ are associated to the gcd of the minors of $D$ (which we already know is associated to the product of the elements on the diagonal). To prove this it suffices to show that these two divide each other, as the proofs in both directions are very similar we only show that the gcd of the minors of order $k$ of $M$ divides the gcd of the minors of order $k$ of $D$.

By definition, $x$ divides $\operatorname{gcd}(\vec{y})$ if and only if $x$ divides every $y$ in $\vec{y}$. So we must show that the gcd of the minors of order $k$ of $M$ divides each minor of order $k$ of the diagonal matrix $D$. Now, there are invertible matrices $P$ and $Q$ such that $P M Q=D$. Hence we must show that $\operatorname{gcd}\left(\vec{m}_{k}\right)$ divides $\operatorname{det}((P M Q)(f, g))$ for all $f$ and $g$. The right-hand side is the determinant of a product of matrices of different sizes whose product is square, which can be simplified with the Binet-Cauchy formula:

$$
\operatorname{det}(M N)=\sum_{\substack{I \in \mathcal{P}(\{1, \ldots, l\}) \\ \#|I|=k}} \operatorname{det}\left(M_{I}\right) \operatorname{det}\left(N_{I}\right)
$$

where $M$ is a $k \times l$ matrix and $N$ is a $l \times k$ matrix. $M_{I}$ (resp. $N_{I}$ ) is the matrix of the $k$ columns (resp. rows) with indices in $I$.

The formalization of this formula builds on the work in [10] and follows Zeng's proof presented in [41]. Note that the standard determinant identity for products of square matrices of the same size follows as a special case of the above formula. Once again the theorem can be expressed compactly using the big operators of SSREFLECT: 


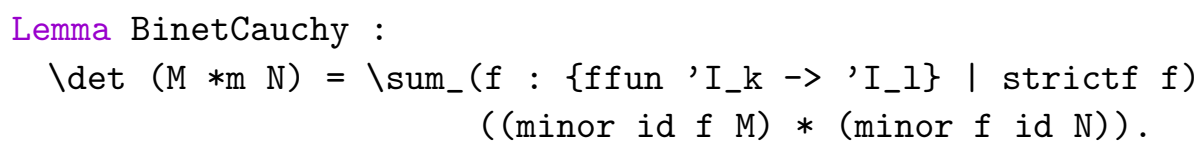

Here the sum is taken over all strictly increasing functions from $\{1, \ldots, k\}$ to $\{1, \ldots, l\}$. We require the functions to be strictly increasing so that the minors that we consider in the sum correspond to the mathematical concept of minor.

This theorem makes it possible for us to transform $\operatorname{det}((P M Q)(f, g))$ to a sum of minors and, once again, it suffices to show that $\operatorname{gcd}\left(\vec{m}_{k}\right)$ divides each of the summands. Hence, after some simplifications, we must show that for all $\mathrm{h}$ and $\mathrm{i}$ we have:

$\backslash$ big[gcdr/0]_f \big[gcdr/0]_g minor f $g$ M \% minor h i M

which is true by definition of the gcd. Note that it is not necessary to require that $f$ and $g$ are strictly increasing. Indeed, if they are not, there are two cases:

- Either $f$ or $g$ is not injective and so minor $f g M=0$.

- If both $f$ and $g$ are injective there exist permutations $r$ and $s$ such that $f{ }^{\prime}=f \backslash 0 \quad r$ and $g^{\prime}=g \backslash 0 \mathrm{~s}$ are strictly increasing. As the permutation of rows or columns of a matrix just leads to the determinant being multiplied by the signature of the permutation we get minor $f \mathrm{~g} M \%=$ minor $f$ ' $g$ ' $M$.

But for all $a$ we have $\operatorname{gcd}(a, 0)=a$ and $\operatorname{gcd}(a, a)=a$, so in each case the terms corresponding to the minors obtained from not strictly increasing $f$ and $g$ does not change the value of the gcd of the minors.

Now if the above result is applied with $k=1$, the uniqueness of the first diagonal element is proved, and then by induction all of the diagonal elements are showed to be unique (up to multiplication by units). This means that for any matrix $M$ equivalent to a diagonal matrix $D$ in Smith normal form, each of the diagonal elements of the Smith normal form of $M$ will be associate to the corresponding diagonal element in $D$. The uniqueness of the Smith normal form is expressed formally as follows:

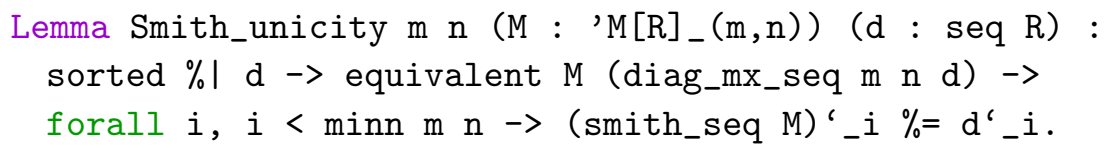

Hence we have proved that the Smith normal form is unique up to multiplication by units. This gives a test to know if two matrices are equivalent. Indeed, since the Smith normal form of a matrix is equivalent to it, two matrices are equivalent if and only if they have the same normal form. Moreover, we know that the decomposition in equation (5.1) is unique up to multiplication by units. Hence we get an algorithm for deciding if two finitely presented modules are isomorphic or not: compute the Smith normal form of the presentation matrices and then test if they are equivalent up to multiplication by units.

This concludes the classification theorem for finitely presented modules over elementary divisor rings. It can be seen as a constructive version of the classification theorem for finitely generated modules over principal ideal domains. Classical proofs of this use the fact that a principal ideal domain $R$ is Noetherian which implies that any $R$-module is coherent, i.e. that any finitely generated module is also finitely presented. But this proof has no computational content (see exercise 3 in chapter III.2 of [30]), so instead we have to restrict to finitely presented modules. In section 4.2 we showed that (constructive) principal ideal domains are elementary divisor rings which gives us the classical result in the case of 
finitely presented modules. In the next section we will prove that more general classes of rings than principal ideal domains are elementary divisor rings which gives more instances of the classification theorem.

\section{Extensions to BÉzout domains that ARE ELEMENTARY DIVISOR RINGS}

As mentioned in the introduction, it is an open problem whether all Bézout domains are elementary divisor rings or not. In order to overcome this, we study different properties that we can extend Bézout domains with to make them elementary divisor rings. The properties we define and discuss in this section are:

(1) Adequacy (i.e. the existence of a gdco operation);

(2) Krull dimension $\leq 1$;

(3) Strict divisibility is well-founded (constructive principal ideal domains).

We have already considered the last one of these in section 4 , but here we formalize an alternative proof that constructive principal ideal domains are elementary divisor rings, using a reduction due to Kaplansky [25]. It consists in first simplifying the problem of computing Smith normal form for $m \times n$ matrices to the $2 \times 2$ case and then showing that any $2 \times 2$ matrix has a Smith normal form if and only if the ring satisfies the "Kaplansky condition". This means that it suffices for us to prove that the three different extensions all imply this condition in order to show that they are elementary divisor rings.

6.1. The Kaplansky condition. The reduction of the computation of Smith normal form of arbitrary $m \times n$ matrices to $2 \times 2$ matrices is done by extracting an algorithm from the proof of theorem 5.1 in [25]. The formalization is done by first implementing this algorithm, called smithmxn, computing the Smith normal form of arbitrary sized matrices assuming an operation computing it for $2 \times 2$ matrices and then proving that this algorithm satisfies smith_spec:

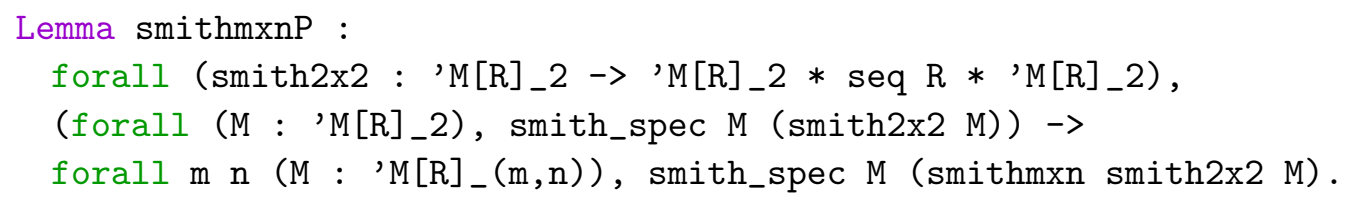

This algorithm has no assumptions on the underlying ring except that it is an integral domain. It can be generalized to arbitrary commutative rings but then we also need to be able to put $1 \times 2$ and $2 \times 1$ matrices in Smith normal form.

Now consider a $2 \times 2$ matrix

$$
\left[\begin{array}{ll}
a & b \\
c & d
\end{array}\right]
$$

with coefficients in a Bézout domain. We can compute $g=\operatorname{gcd}(a, c)$ and $a_{1}$ and $c_{1}$ such that $a=a_{1} g$ and $c=c_{1} g$. We also have $u$ and $v$ such that $u a_{1}+v c_{1}=1$. Using this we can form:

$$
\begin{aligned}
{\left[\begin{array}{cc}
u & v \\
-c_{1} & a_{1}
\end{array}\right]\left[\begin{array}{ll}
a & b \\
c & d
\end{array}\right] } & =\left[\begin{array}{cc}
u a+v c & u b+v d \\
-c_{1} a+a_{1} c & -c_{1} b+a_{1} d
\end{array}\right] \\
& =\left[\begin{array}{cc}
u a+v c & u b+v d \\
0 & -c_{1} b+a_{1} d
\end{array}\right]
\end{aligned}
$$


So it suffices to consider matrices of the following shape:

$$
\left[\begin{array}{ll}
a & b \\
0 & c
\end{array}\right]
$$

and without loss of generality we can assume that $\operatorname{gcd}(a, b, c)=1$. Now, such a matrix has a Smith normal form if and only if it satisfies the Kaplansky condition: for all $a, b, c \in R$ with $\operatorname{gcd}(a, b, c)=1$ there exist $p, q \in R$ with $\operatorname{gcd}(p a, p b+q c)=1$.

The interesting step for the reduction is the right to left direction of the "if and only if", so let us sketch how it is proved: assume that $R$ is a Bézout domain that satisfies the Kaplansky condition and consider an upper triangular matrix with elements $a, b$ and $c$ with $\operatorname{gcd}(a, b, c)=1$. From the Kaplansky condition we get $p$ and $q$ such that $\operatorname{gcd}(p a, p b+q c)=1$. This means that we also have $x_{1}$ and $y_{1}$ such that $p a x_{1}+(p b+q c) y_{1}=1$. By reorganizing this we get $p\left(a x_{1}+b y_{1}\right)+q c y_{1}=1$, let $x=a x_{1}+b y_{1}$ and $y=c y_{1}$. We can form the product:

$$
\left[\begin{array}{cc}
p & q \\
-y & x
\end{array}\right]\left[\begin{array}{ll}
a & b \\
0 & c
\end{array}\right]\left[\begin{array}{cc}
x_{1} & p b+q c \\
y_{1} & -p a
\end{array}\right]=\left[\begin{array}{cc}
1 & 0 \\
0 & -a c
\end{array}\right]
$$

In order to formalize this proof we assume that we have an operation taking $a, b$ and $c$ computing $p$ and $q$ satisfying the Kaplansky condition:

Variable kap : $\mathrm{R} \rightarrow \mathrm{R} \rightarrow \mathrm{R} \rightarrow \mathrm{R} * \mathrm{R}$.

Hypothesis kapP : forall (a b c : R), gcdr a (gcdr b c) \%=1 $\rightarrow$

let: $(p, q):=$ kap a b c in coprimer $(p * a)(p * b+q * c)$.

We then define a function kapw $: \mathrm{R} \rightarrow \mathrm{R} \rightarrow \mathrm{R} \rightarrow \mathrm{R} * \mathrm{R}$ to extract the two witnesses $x_{1}$ and $y_{1}$ from above, i.e. $x_{1}$ and $y_{1}$ such that $x_{1} p a+y_{1}(p b+q c)=1$. To do this we first prove:

Lemma coprimerP ( $\mathrm{a} b: \mathrm{R})$ :

reflect (exists (xy : R $* \mathrm{R}$ ), xy.1*a +xy.2*b=1) (coprimer a b).

and we can then define a function computing $\left(x_{1}, y_{1}\right)$ by turning the existential statement in coprimerP into a $\Sigma$-type (i.e. a dependent pair). More precisely, we have defined it by:

Definition kapW a b c : $\mathrm{R} * \mathrm{R}:=$

let: $(p, q):=\operatorname{kap} a b c$ in

if coprimerP $(\mathrm{p} * \mathrm{a})(\mathrm{p} * \mathrm{~b}+\mathrm{q} * \mathrm{c})$ is ReflectT $\mathrm{P}$

then projT1 (sig_eqW P) else $(0,0)$.

Here sig_eqW is a function from the SSREFLECT library that transforms our existential statement into a $\Sigma$-type, the first component of the resulting $\Sigma$-type is then extracted using projT1. This is possible because $\mathrm{R}$ is taken to be an SSREFLECT "choice type", i.e. a type with a choice operator.

Once we have defined kapw, we can easily write the function computing Smith normal form of $2 \times 2$ matrices, called kap_smith, and prove that it satisfies smith_spec:

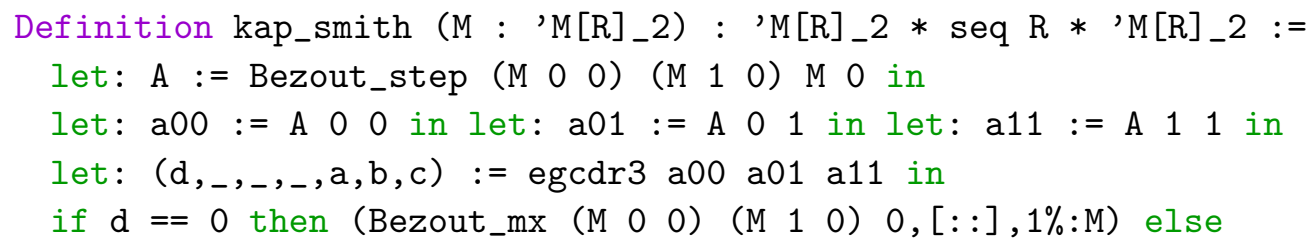




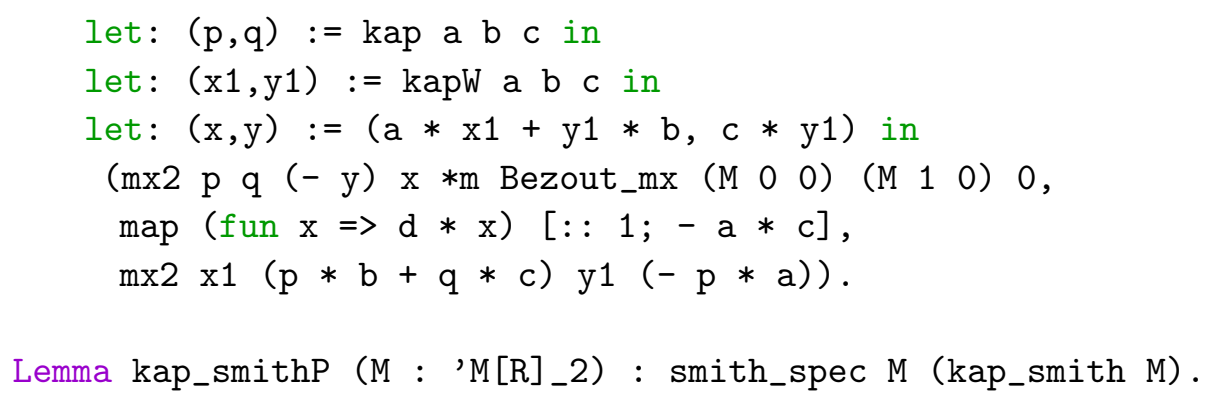

Here $\mathrm{mx} 2$ is a notation to define $2 \times 2$ matrices and egcdr 3 computes the Bézout coefficients for 3 elements.

We have also formalized the other direction, so for a Bézout domain, satisfying the Kaplansky condition is equivalent to being an elementary divisor ring. Hence it suffices to prove that the various extensions to Bézout domains satisfy the Kaplansky condition in order to get that they are elementary divisor rings.

6.2. The three extensions to Bézout domains. In this section we discuss three extensions to Bézout domains that imply the Kaplansky condition.

6.2.1. Adequate domains. In [22] Helmer introduced the notion of adequate domains. These are Bézout domains where for any $a, b \in R$, with $b \neq 0$, there exists $r \in R$ such that:

(1) $r \mid b$,

(2) $r$ is coprime with $a$, and

(3) for all non unit $d$ such that $d r \mid b$ we have that $d$ is not coprime with $a$.

We have proved that this notion is equivalent to having a "gdco" function. This function has previously been introduced by one of the authors in [6] in order to implement quantifier elimination for algebraically closed fields. It has also other applications in algebra, see [28]. It takes two elements $a, b \in R$, with $b \neq 0$, and computes $r$ such that:

(1) $r \mid b$,

(2) $r$ is coprime with $a$, and

(3) for all divisors $d$ of $b$ that is coprime to $a$ we have $d \mid r$.

This means that $r$ is the greatest divisor of $b$ that is coprime to $a$. These notions are expressed in CoQ as:

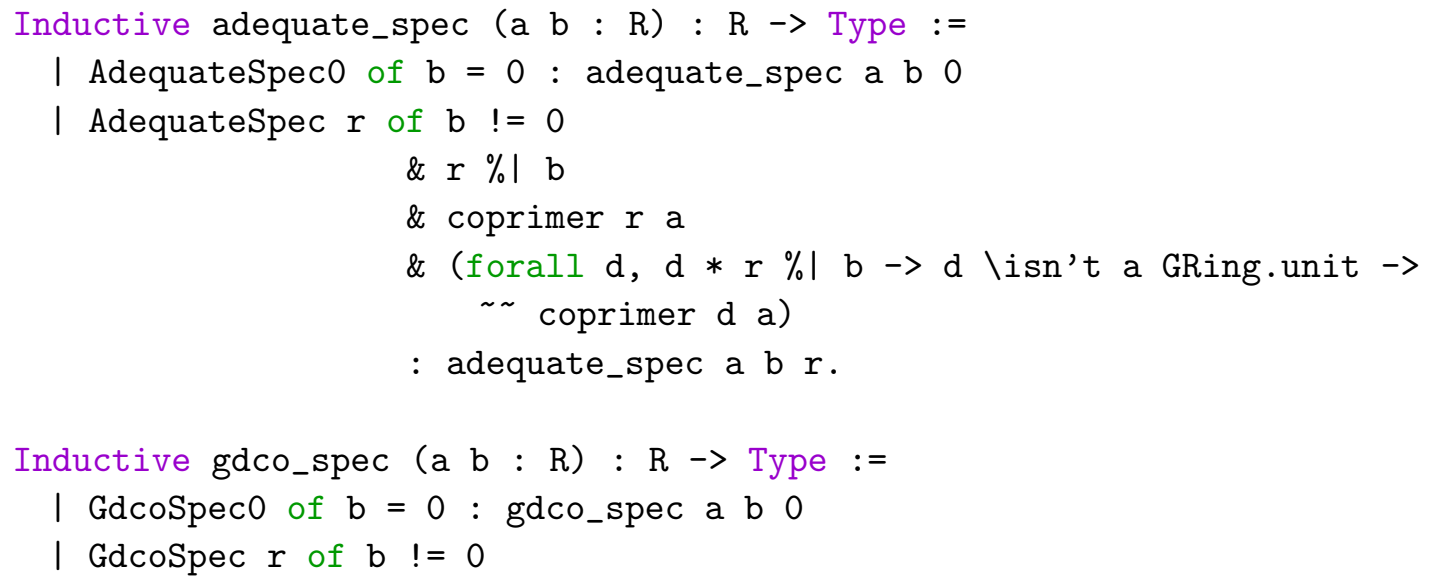




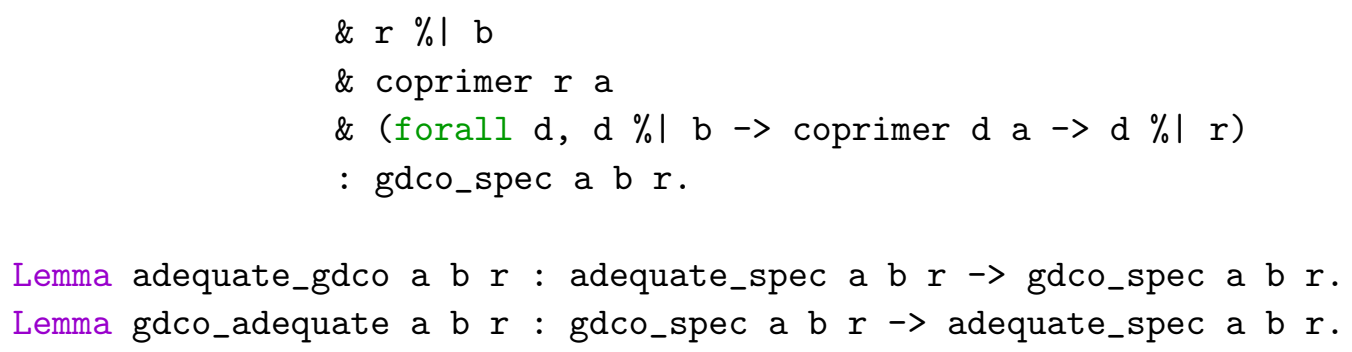

We have implemented an algorithm called gdco_kap that computes $p$ and $q$ in the Kaplansky condition using the gdco operation. Using this we have proved:

Lemma gdco_kapP (a b c : R) : gcdr a (gcdr b c) \%=1 $\rightarrow$

let: $(p, q):=$ gdco_kap a b c in coprimer $(p * a)(p * b+q * c)$.

Using this we can define a function that computes the Smith normal form for any matrix over an adequate domain:

Definition gdco_smith $:=$ smithmxn (kap_smith gdco_kap).

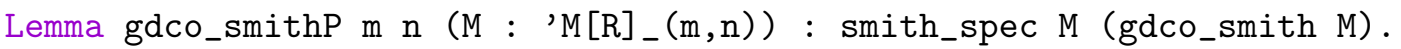

Hence we get that adequate domains are elementary divisor rings.

6.2.2. Krull dimension $\leq 1$. The next class of rings we study are Bézout domains of Krull dimension $\leq 1$. Classically Krull dimension is defined as the supremum of the length of all chains of prime ideals, this means that a ring has Krull dimension $n \in \mathbb{N}$ if there is a chain of prime ideals:

$$
\mathfrak{p}_{0} \subsetneq \mathfrak{p}_{1} \subsetneq \cdots \subsetneq \mathfrak{p}_{n}
$$

but no such chain of length $n+1$. For example, a field has Krull dimension 0 and any principal ideal domain (that is not a field) has Krull dimension 1. This can be defined constructively using an inductive definition as in [26]. Concretely an integral domain $R$ is of Krull dimension $\leq 1$ if for any $a, u \in R$ there exists $v \in R$ and $n \in \mathbb{N}$ such that

$$
a \mid u^{n}(1-u v)
$$

In order to prove that Bézout domains of Krull dimension $\leq 1$ are adequate we first prove:

Hypothesis krull1 : forall a $u$, exists $m v, a \% l u^{-}+m *(1-u * v)$.

Lemma krull1_factor a $\mathrm{b}$ : exists $\mathrm{n}$ b1 b2,

$\left[\& \& 0<\mathrm{n}, \mathrm{b}==\mathrm{b} 1 * \mathrm{~b} 2\right.$, coprimer $\left.\mathrm{b} 1 \mathrm{a} \& \mathrm{~b} 2 \% \mathrm{a}{ }^{-}+\mathrm{n}\right]$.

This means that given $a$ and $b$ we can compute $n \in \mathbb{N}$ and $b_{1}, b_{2} \in R$ such that $n \neq 0$, $b=b_{1} b_{2}, b_{1}$ is coprime with $a$ and $b_{2} \mid a^{n}$. If we set $r$ to $b_{1}$ in the definition of adequate domains we have to prove:

(1) $b_{1} \mid b_{1} b_{2}$,

(2) $b_{1}$ is coprime with $a$, and

(3) for all non unit $d$ such that $d b_{1} \mid b_{1} b_{2}$ we have that $d$ is not coprime with $a$. 
The first two are obvious. For the third point, we have to prove that any non-unit $d$ that divides $b_{2}$ is not coprime with $a$. So it suffices to prove that any $d$ coprime with $a$ that divides $b_{2}$ is a unit. Now as $n \neq 0$ we get that $d$ is coprime with $a^{n}$, but $d \mid b_{2}$ and $b_{2} \mid a^{n}$ so $d$ must be a unit. We have formalized this argument in:

Lemma krull1_adequate $\mathrm{a} b:\{\mathrm{r}: \mathrm{R}$ \& adequate_spec a $\mathrm{b} \mathrm{r}\}$.

This means that Bézout domains of Krull dimension $\leq 1$ are adequate and hence satisfy the Kaplansky condition, which in turn means that they are elementary divisor rings:

Definition krull1_gdco a b := projT1 (krull1_adequate a b).

Definition krull1_smith := gdco_smith krull1_gdco.

Lemma krul11_smithP m $\mathrm{n}\left(\mathrm{M}:{ }^{\prime} M[R]_{-}(\mathrm{m}, \mathrm{n})\right)$ : smith_spec $M$ (krull1_smith $\left.M\right)$.

6.2.3. Constructive principal ideal domains. Finally, we have showed that constructive principal ideal domains are adequate domains by proving that given $a$ and $b$ we can compute $r$ satisfying gdco_spec:

Lemma pid_gdco ( $R:$ pidType) ( $a b: R):\{r: R \&$ gdco_spec $a b r\}$.

The construction of the greatest divisor of $a$ coprime to $b$ in a constructive principal ideal domain is done as in the particular case of polynomials in [6]. If $\operatorname{gcd}(a, b)$ is a unit, then $a$ is trivially the result, otherwise we get $a^{\prime}$ by dividing $a$ by $\operatorname{gcd}(a, b)$ and we repeat the process with $a^{\prime}$ and $b$. This process terminates because when $\operatorname{gcd}(a, b)$ is not a unit, $a^{\prime}$ strictly divides $a$ and by our definition of constructive principal ideal domains, there cannot be an infinite decreasing sequence for strict divisibility.

This way we get an alternative proof that constructive principal ideal domains are elementary divisor rings:

Definition pid_smith $:=$ gdco_smith (fun a b $=>$ projT1 (pid_gdco a b)).

Lemma pid_smithP $m \mathrm{n}\left(\mathrm{M}:{ }^{\prime} \mathrm{M}[\mathrm{R}]_{-}(\mathrm{m}, \mathrm{n})\right)$ : smith_spec $M$ (pid_smith $\left.M\right)$.

This proof is simpler in the sense that we first reduce the problem of computing the Smith normal form to computing the gdco of two elements. This way, the part of the proof based on well-founded recursion is concentrated to pid_gdco instead of being interleaved in the algorithm computing the Smith normal form of arbitrary $m \times n$ matrices.

\section{RELATED WORK}

Most proof systems have one or more libraries of formalized linear algebra. However, the specificity of our work is that it is more general than the usual study of vector spaces (we do not require scalars to be in a field, but only in an elementary divisor ring) while still retaining an algorithmic basis, as opposed to a purely abstract and axiomatized development. In particular, this work constitutes to our knowledge the first formal verification of an algorithm for the Smith normal form of matrices.

A fair amount of module theory and linear algebra has been formalized [35] in MizAR. But it is based on classical logic and does not account for underlying algorithmic aspects. 
Likewise, a HOL Light library [20] proves significant results in linear algebra and on the topology of vector spaces, but it is specialized to $\mathbb{R}^{n}$ and also classical.

Some other developments focus more on the algebra of vectors and matrices, without providing support for point-free reasoning on subspaces. Let us cite 32 in IsABELLE, which aims primarily to certify linear inequalities and [15, 23] in ACL2, formalizing only matrix algebra.

In CoQ too, older developments focus on the representation of matrices like [29], or classical linear algebra over a field like [36], based on [34]. One exception is of course the more recent work [17] we already mentioned and on which we based this work, extending it from finitely generated vector spaces to finitely presented modules over elementary divisor rings.

The authors are also developing a library of computational algebra called COQEAL the CoQ Effective Algebra Library [5, 12]. It contains many examples of algorithms from linear algebra like the rank of matrices over fields and Strassen's matrix multiplication [12], the Sasaki-Murao algorithm for computing the characteristic polynomial of a matrix over a commutative ring [10, and the kernel of a matrix over a field [24].

Two of the authors have previously formalized the theory of finitely presented modules in CoQ [7, building on a previous formalization of coherent and strongly discrete rings [9] that provides a basis for a general treatment of matrix algebra. The present work extends this to the theory of finitely presented modules over elementary divisor rings, which gives a means for deciding whether two finitely presented modules are isomorphic or not as described in section 5.3. It also provides concrete instances solving the basic algorithmic problems underlying the work on finitely presented modules as elementary divisor rings provides interesting examples of coherent strongly discrete rings.

There has also been a lot of work on implementing algorithms for computing the Smith normal form over various rings in computer algebra systems like AXIOM, MAPLE and MAGMA. There are also lots of literature on very efficient algorithms for computing the Smith normal form over various coefficient rings, see for instance [13, 37, 38, 39, 40]. The motivation behind this kind of work is however different from the work presented here as the focus is on devising very efficient algorithms without formal proofs of correctness. Because of these the focus is on specific coefficient rings and not on full generality. However, using the CoQEAL approach we expect that it would be both possible and very interesting to extend this work to also implement more efficient algorithms from computer algebra.

\section{Conclusions And Future WORK}

The relationships between the notions introduced in this paper are depicted in figure 1 . The numbers on the edges denote the sections in which the different implications and inclusions are proved:

The arrow between PID and Krull dimension $\leq 1$ is dashed because it has not been formally proved yet. A constructive proof of this can be found in [26]. We currently see two options to formalize it: either we try to develop more extensively the theory of ideals to stick close to the paper proof, or we expand statements on ideal to statements on elements. Unlike the former, the latter option would require no further infrastructure, but it is likely that the size of the proof would explode, as in some proofs where we already had to talk about elements instead of ideals (e.g. the lemma krull1_factor in the current state of the formalization). 


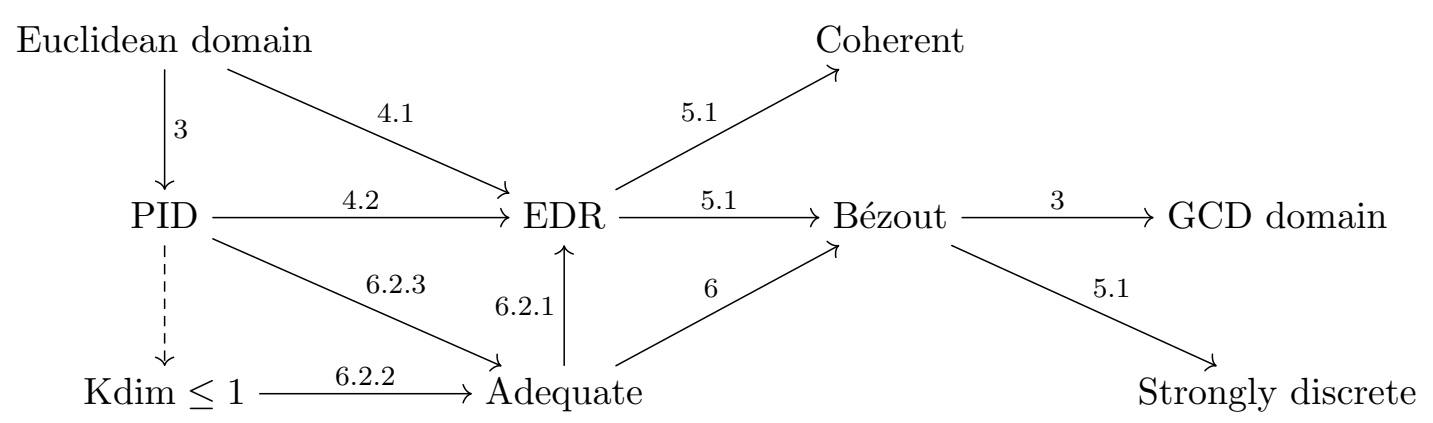

Figure 1: Relationship between the defined notions

It has been mentioned that $\mathbb{Z}$ and $k[x]$ where $k$ is a field are the basic examples for all of these rings. Many more examples of Bézout domains are presented in the chapters on Bézout domains and elementary divisor rings in [14] (for instance, Bézout domains of arbitrary finite Krull dimension and an example of a Bézout domain that is not adequate). It would be interesting see which of these could be done in a constructive setting and formalize them in order to get more instances than $\mathbb{Z}$ and $k[x]$.

Note that the Kaplansky condition in section 6 is expressed using first-order logic. It means that the open problem whether all Bézout domains are elementary divisor rings can be expressed using first-order logic. We have formulated the problem this way and applied various automatic theorem provers in order to try to find a proof that Bézout domains, alone, and with the two other assumptions (adequacy or Krull dimension $\leq 1$ ) are elementary divisor rings. However, none managed so far.

We have in this paper presented the formalization of many results on elementary divisor rings. This way we get interesting examples of coherent strongly discrete rings and concrete algorithms for studying finitely presented modules. All of the proofs have been performed in a constructive setting, and except for principal ideal domains, without chain conditions.

The size of the companion material is approximately 9000 lines of code.

An important application of this work would be to compute the homology of chain complexes which provides a means to study properties of mathematical objects like topological spaces. By computing homology one associates modules to these kinds of objects, giving a way to distinguish between them. The Smith normal form of matrices with coefficients in the ring of integers (denoted by $\mathbb{Z}$ ) is at the heart of the computation of homology as the universal coefficient theorem for homology [21] states that homology with coefficients in $\mathbb{Z}$ determines homology with coefficients in any other abelian group. By developing the theory for more general rings than $\mathbb{Z}$ it should be possible to implement and reason about other functors from homological algebra as well, like for instance cohomology and the Ext and Tor functors.

\section{ACKNOWLEDGMENTS}

The authors would like to thank Thierry Coquand and Henri Lombardi for interesting discussions. The authors are also grateful to Dan Rosén and Jean-Christophe Filliâtre for 
helping us explore the Kaplansky condition using various automatic theorem provers. We would also like to thank Claire Tête for useful comments on a preliminary version of the paper. Finally we would like to thank the anonymous reviewers for their helpful remarks.

\section{REFERENCES}

[1] M. Barakat and M. Lange-Hegermann. An Axiomatic Setup for Algorithmic Homological Algebra and an Alternative Approach to Localization Journal of Algebra and Its Applications, 10(2):269-293, 2011.

[2] M. Barakat and D. Robertz. HOMALG - A Meta-Package for Homological Algebra. Journal of Algebra and Its Applications, 7(3):299-317, 2008.

[3] Y. Bertot, G. Gonthier, S. Biha, and I. Pasca. Canonical big operators. In Theorem Proving in HigherOrder Logics (TPHOLs'08), volume 5170 of LNCS, pages 86-101, 2008.

[4] G. Cano and M. Dénès. Matrices à blocs et en forme canonique In D. Pous and C. Tasson, editors, JFLA - Journées francophones des langages applicatifs, Aussois, France, 2013. Damien Pous and Christine Tasson, Damien Pous and Christine Tasson.

[5] C. Cohen, M. Dénès, and A. Mörtberg. Refinements for Free! In G. Gonthier and M. Norrish, editors, Certified Programs and Proofs, volume 8307 of Lecture Notes in Computer Science, pages 147-162. Springer International Publishing, 2013.

[6] C. Cohen and A. Mahboubi. A formal quantifier elimination for algebraically closed fields In Proceedings of the 10th ASIC and 9th MKM international conference, and 17th Calculemus conference on Intelligent computer mathematics, AISC'10/MKM'10/Calculemus'10, pages 189-203, Berlin, Heidelberg, 2010. Springer-Verlag.

[7] C. Cohen and A. Mörtberg. A Coq Formalization of Finitely Presented Modules. In Interactive Theorem Proving - 5th International Conference, ITP 2014, Vienna, Austria., pages 193-208. Springer, 2014.

[8] CoQ development team. The CoQ Proof Assistant Reference Manual, version 8.4. Technical report, Inria, 2012.

[9] T. Coquand, A. Mörtberg, and V. Siles. Coherent and Strongly Discrete Rings in Type Theory. In C. Hawblitzel and D. Miller, editors, Certified Programs and Proofs, volume 7679 of Lecture Notes in Computer Science, pages 273-288. Springer Berlin Heidelberg, 2012.

[10] T. Coquand, A. Mörtberg, and V. Siles. A formal proof of Sasaki-Murao algorithm. Journal of Formalized Reasoning, 5(1), 2013.

[11] W. Decker and C. Lossen. Computing in Algebraic Geometry: A Quick Start using SINGULAR. Springer Publishing Company, Incorporated, 2006.

[12] M. Dénès, A. Mörtberg, and V. Siles. A Refinement-Based Approach to Computational Algebra in Coq. In L. Beringer and A. Felty, editors, Interactive Theorem Proving, volume 7406 of Lecture Notes in Computer Science, pages 83-98. Springer Berlin Heidelberg, 2012.

[13] J.-G. Dumas, B. D. Saunders, and G. Villard. On Efficient Sparse Integer Matrix Smith Normal Form Computations, Journal of Symbolic Computation, 32(1-2):71-99, 2001.

[14] L. Fuchs and L. Salce. Modules Over Non-Noetherian Domains Mathematical surveys and monographs. American Mathematical Society, 2001.

[15] R. Gamboa, J. Cowles, and J. V. Baalen. Using ACL2 arrays to formalize matrix algebra. In Fourth International Workshop on the ACL2 Theorem Prover and Its Applications (ACL2 '03), July 2003.

[16] F. Garillot, G. Gonthier, A. Mahboubi, and L. Rideau. Packaging mathematical structures In Proceedings 22nd International Conference on Theorem Proving in Higher Order Logics (TPHOLs'09), volume 5674 of $L N C S$, pages 327-342, 2009.

[17] G. Gonthier. Point-Free, Set-Free Concrete Linear Algebra. In Interactive Theorem Proving, volume 6898 of $L N C S$, pages 103-118. Springer-Verlag, 2011.

[18] G. Gonthier and A. Mahboubi. A Small Scale Reflection Extension for the Coq system. Technical report, Microsoft Research INRIA, 2009.

[19] G.-M. Greuel and G. Pfister. A Singular Introduction to Commutative Algebra. Springer Publishing Company, Incorporated, 2nd edition, 2007.

[20] J. Harrison. The HOL light theory of euclidean space J. Autom. Reasoning, 50(2):173-190, 2013.

[21] A. Hatcher. Algebraic Topology. Cambridge University Press, 1st edition, 2001. 
[22] O. Helmer. The elementary divisor theorem for certain rings without chain condition. Bulletin of the American Mathematical Society, 49:225-236, 1943.

[23] J. Hendrix. Matrices in ACL2. In Fourth International Workshop on the ACL2 Theorem Prover and Its Applications (ACL2 '03), July 2003.

[24] J. Heras, T. Coquand, A. Mörtberg, and V. Siles. Computing Persistent Homology Within Coq/SSReflect. ACM Transactions on Computational Logic, 14(4):1-26, November 2013.

[25] I. Kaplansky. Elementary divisors and modules. Transactions of the American Mathematical Society, 66:464-491, 1949.

[26] H. Lombardi and C. Quitté. Algèbre commutative, Méthodes constructives: Modules projectifs de type fini. Calvage et Mounet, 2011.

[27] D. Lorenzini. On Bézout Domains. http://www.math.uga.edu/ lorenz/Bezout.pdf

[28] H. Lüneburg. On a Little but Useful Algorithm. In Proceedings of the 3rd International Conference on Algebraic Algorithms and Error-Correcting Codes, AAECC-3, pages 296-301, London, UK, UK, 1986. Springer-Verlag.

[29] N. Magaud. Programming with Dependent Types in Coq: a Study of Square Matrices, Jan 2005. Unpublished. A preliminary version appeared in Coq contributions.

[30] R. Mines, F. Richman, and W. Ruitenburg. A Course in Constructive Algebra. Springer-Verlag, 1988.

[31] B. Nordström. Terminating general recursion. BIT, 28(3):605-619, 1988.

[32] S. Obua. Proving bounds for real linear programs in isabelle/hol volume 3603 of Lecture Notes in Computer Science, pages 227-244. Springer, 2005.

[33] H. Perdry. Strongly Noetherian rings and constructive ideal theory. Journal of Symbolic Computation, $37(4): 511-535,2004$.

[34] L. Pottier. User contributions in Coq: Algebra, 1999.

[35] P. Rudnicki, C. Schwarzweller, and A. Trybulec. Commutative algebra in the mizar system, J. Symb. Comput., 32(1/2):143-169, 2001.

[36] J. Stein. Documentation of my formalization of linear algebra. Technical report, 2001.

[37] A. Storjohann. Near Optimal Algorithms for Computing Smith Normal Forms of Integer Matrices. In Proceedings of the 1996 International Symposium on Symbolic and Algebraic Computation, ISSAC '96, pages 267-274, New York, NY, USA, 1996. ACM.

[38] A. Storjohann. Computing Hermite and Smith normal forms of triangular integer matrices. Linear Algebra and its Applications, 282(1-3):25-45, 1998.

[39] A. Storjohann and G. Labahn. A fast Las Vegas algorithm for computing the Smith normal form of a polynomial matrix. Linear Algebra and its Applications, 253(1-3):155-173, 1997.

[40] G. Villard. Generalized Subresultants for Computing the Smith Normal Form of Polynomial Matrices Journal of Symbolic Computation, 20(3):269-286, 1995.

[41] J. Zeng. A bijective proof of Muir's identity and the Cauchy-Binet formula Linear Algebra and its Applications, 184(0):79-82, 1993.

This work is licensed under the Creative Commons Attribution-NoDerivs License. To view a copy of this license, visit http://creativecommons.org/licenses/by-nd/2.0/ or send a letter to Creative Commons, 171 Second St, Suite 300, San Francisco, CA 94105, USA, or Eisenacher Strasse 2, 10777 Berlin, Germany 